\title{
Beiträge zur Kenntnis der Reproduktions- und Regene- rationsvorgänge bei Pilzen und der Bedingungen des Absterbens myzelialer Zellen von Aspergillus niger.
}

Von Paul Köhler.

Mit 10 Abbildungen im Texte.

\section{A. Einleitung und Literaturangabe.}

Reproduktion und Regeneration sind Ersatztätigkeiten, die man an höheren Pflanzen schon seit altersher kennt, praktlich ausgenützt und auch schon oft wissenschaftlich untersucht hat. Unter den niederen Pflanzen sind in dieser Hinsicht sehr eingehend Moose und in jüngster Zeit vor allem die Algen bearbeitet worden. Was die Pilze anbetrifft, so beschränken sich die Kenntnisse über ihre Reproduktions- und Regenerationsfähigkeit teils auf Tatsachen, die mehr nebenbei in Anschluß an andere Arbeiten gefunden wurden, teils auf gelegentliche Beobachtungen und Untersuchungen. Der Umfang der für diese Organismen gefundenen Ergebnisse ist infolgedessen noch klein. Den Nachweis davon soll im folgenden eine Zusammenstellung der bis jetzt bekannten Resultate geben.

Von manchen Schimmelpilzen vermag eine jede ausgewachsene Zelle des vegetativen Myzels einen ganzen Organismus zu reproduzieren²). Ebenso entsteht aus einer abgetrennten vegetativen Hyphe von Mucor, die ihre Wunde durch Bildung einer Membran verschließt, ein vollständiger Organismus ${ }^{3}$ ). Die Untersuchungen von Klebs an Eurotium herbariorum lehren, daß auch differenziertere Zellen ebenso reproduktionsfähig sein können wie vegetative Zellen Denn die Konidienträger dieses Pilzes lassen, wenn sie in Nährlösung untergetaucht werden, ein vegetatives Myzel hervorgehen ${ }^{4}$ ).

Auch Parasiten können Reproduktionsvorgänge auslösen. So treibt nach Brefeld ein Sporangiumträger von Mucor Mucedo, der von Pipto-

1) Unter Reproduktion verstehe ich in Anlehnung an Pfeffer (Pflanzenphysiologie, Leipzig 1901, Bd. II, pag. 204) einen Ersatz durch Neubildungen, Auswachsen von Anlagen usw., unter Regeneration dagegen die Ersatztätigkeit, die eine Wiederherstellung des Hinweggenommenen von der Wundfläche aus bewirkt.

2) Pfeffer, Pflanzenphys., Leipzig, Bd. II, pag. 205.

3) van Tieghem, Annal. d. scienc. naturell. 1875, VI. sér., T. I, pag. 19.

4) Klebs, Die Bedingungen der Fortpflanzung bei einigen Algen und Pilzen, 1896, pag. 458 . 
cephalis befallen wird, Seitenzweige, die ebenfalls zu Sporangienträgern werden, indem an ihren Enden kleine Sporangien, entstehen ${ }^{1}$ ):

Wie die untergetauchten Konidienträger von Eurotium herbariorum verhalten sich auch Teilstücke von Fruchtkörpern einiger höherer Pilze. Brefeld brachte Teile des Fruchtkörpers von Coprinus stercorarius ${ }^{2}$ ), Coprinus ephemerus ${ }^{3}$ ) und Peziza Sklerotiorum ${ }^{4}$ ) in Nährlösung oder auf Nährsubstrat und sah, wie aus ihnen ein vegetatives Myzel hervorging. Es war gleichgültig, ob er zu diesen Versuchen Stielteile, Hutteile oder Lamellen verwendete: stets erhielt er dasselbe Resultat.

Die gleiche Fähigkeit wie diese Teilstücke zeigen nach de Bary die Sklerotien der Sklerotinien ${ }^{5}$ ); denn diese lassen, wenn sie sich innerhalb einer Nährlösung befinden, die an die Schnittfläche grenzenden Hyphen zu einem vegetativen Myzel austreiben. In einem feuchten Raume dagegen entsteht bei denselben Sklerotien, wie auch an dem Sklerotium von Claviceps purpurea eine kallusartige Wucherung auf der Schnittfläche.

Ähnliches Verhalten beobachtete Brefeld an den Sklerotien von Coprinus stercorarius, insofern bei diesen die Zellen der Schnittfläche auf Nährsubstrat zu Myzel auswachsen ${ }^{6}$ ). Auf feuchtem Sande aber verwandeln sich die durch den Schnitt frei gelegten Zellen des Sklerotiums in Rindenzellen, indem sich ihre Membran schwärzt und außerdem kutilarisiert wird ${ }^{7}$ ).

Dieselbe Abhängigkeit in der Qualität der Neubildung von dem sie umgebenden Medium zeigen die von Brefeld untersuchten Teilstücke des Fruchtkörpers von Coprinus, die, wie bereits erwähnt wurde, in Nährlösung zu Myzel aussprossen; denn sie produzieren auf feuchtem Sande ausgelegt, kleine, aber sonst normale Fruchtkörper mit Hut und Stiel $^{8}$ ). Hierbei ist es wieder gleich, welchem Gewebe des Fruchtkörpers die Teilstücke entstammen.

Bei Coprinus stercorarius konnte Brefeld auch sehen, wie der weggeschnittene Hut von dem noch wachsenden Fruchtträger regeneriert wird $\left.{ }^{9}\right)$.

1) Brefeld, Untersuchungen über Schimmelpilze, 1872, Heft I, Tafel I, Fig. 24. Beschreibung pag. 58.

2) Ders., Ibidem, 1877, Heft III, pag. 78 ff.

3) Ders., Ibidem, Heft III, pag. 114.

4) Ders., Ibidem, Heft IV, pag. 121, Anm.

5) de Bary, Morphologie und Biologie der Pilze, 1884, pag. 42.

6) Brefeld, l. c. Heft III, pag. 82.

7) Ders., l. c. Heft III, pag. 25.

8) Ders., l. c. Heft III, pag. $72 \mathrm{ff}$.

9) Ders., l. c. Heft III, pag. 69. 
Die Reproduktion von Fruchtkörpern am Hut oder Stiel ist nun ein Verhalten, wie es auch bei anderen höheren Pilzen festgestellt worden ist. - Neue Fruchtkörper bildet nach Goebel ${ }^{1}$ ) der Fruchtkörper von Stereum hirsutum, wenn er beschnitten, aber auf seinem natürlichen Nährboden belassen wird, und zwar gehen die Neubildungen von den Schnittflächen aus.

Werden die „Zarten Spitzen“ einer von Hennings beobachteten Xylaria, die der Gattung Kretschmeria angehört, von Nacktschnecken abgenagt, so entwickeln sich auf den zurückbleibenden Stielstümpfen kopfförmige Fruchtkörper ${ }^{2}$ ). Eine ähnliche Reaktion konstatierte Hennings an dem Fruchtstiel von Claviceps purpurea, der im Frühjahr aus dem Sklerotium hervorbricht. Wird nämlich das Köpfchen, in dem die Askussporen entstehen, abgebrochen, so reproduziert der Stiel von neuem ,seitlich halbkugelige Köpfchen“" ${ }^{3}$ ).

Hennings führt weiter eine Anzahl von Pilzen an, auf deren Hut aus unbekannten Ursachen kleinere Fruchtkörper mit Stiel und Hut hervorwachsen ${ }^{4}$ ). Nach seinen Angaben sollen derartige Abnormitäten in der Natur öfter vorkommen, z. B. bei Boletus scaber, Boletus subtomentosus, Russula emetica, Tricholoma rutilans, Tricholoma breviceps usw.

Endlich sind auch manche Pilze imstande, Schäden, die sie durch Verletzungen erhalten haben, zu verheilen. Bei Boletus edulis wird nach Massart das Hymenium dort, wo es von Schnecken weggefressen worden ist, von neuem gebildet ${ }^{5}$ ). Bei Trametes gibbosus entstand auf der Schnittfäche, die von Massart senkreeht zu den horizontalen Schichten des Fruchtkörpers gelegt wurde, eine nene Oberfläche, die genau so stark war als die, die in derselben Zeit zu der unberührten oberen Seite hinzukam ${ }^{6}$ ). Endlich beobachtete Massart, daß an Agaricus campestris spontane Verletzungen vollkommen vernarbt werden ${ }^{7}$ ). Daß Agaricus campestris überhaupt ein besonders günstiges Objekt für das Studium der Reproduktion sowohl als auch der Regeneration ist, beweisen die vorläufigen Mitteilungen von Magnus, der z. B. eine Regeneration des Hymeniums und der Lamellen an diesem Pilze beobachtete ${ }^{8}$.

1) Goebel, Flora 1903, pag. $143 \mathrm{ff}$.

2) P. Hennings. Hedwigia, XL, 1901, pag. 140.

3) Ders., l. c. pag. 140.

4) Ders., l. c. pag. 138.

5) Massart, La cicatrisation chez les régétaux, 1898, pag. 18.

6) Ders., l. c. pag. 19.

7) Ders., l. c. pag. 18.

8) W. Magnus, Ber. d. botan. Gesellsch. 1903, pag. 129. 
Damit ist in großen Umrissen alles das angegeben, was wir bis jetzt über Reproduktion und Regeneration bei Pilzen wissen. Es lag nahe, diese Reaktionsfähigkeit der Pilze einmal einer systematischen Bearbeitung zu unterziehen, um durch Ausfüllung von Lücken und durch Heranziehung von noch nicht untersuchten Objekten das Erfahrungsmaterial über diesen Gegenstand zu vermehren. $\mathrm{Zu}$ diesem Zwecke wurde eine Reihe von Pilzen, von den einfachsten bis zu den differenziertesten Formen, auf ihre Reproduktions- und Regenerationsfähigkeit geprüft.

Die durch diese Untersuchungen gewonnenen Resultate ermöglichen einen geschlossenen Überblick über die betreffenden Erscheinungen und gestatten uns weiterhin, zur Diskussion einiger Fragen allgemeinerer Natur zu schreiten. Hierher gehört in erster Linie die Frage nach der Allgemeinbefähigung der einzelnen Zellen und Zellteile der untersuchten Pilze und deren Realisierbarkeit. Daran schließt sich als zweite Frage, in welcher Weise die Außenbedingungen auf die Lenkung der Reproduktionstätigkeit von Einfluß sind, und damit im Zusammenhange eine dritte Frage, inwieweit nämlich die Qualität der Neubildung vom Zustande des Teilstückes abhängig ist.

\section{B. Experimenteller Teil.}

\section{Versuche mit Schimmelpilzen.}

Die Untersuchungen wurden zuerst an den einzelligen Schimmelpilzen Mucor stolonifer und Phycomyces nitens vorgenommen. Was das vegetative Myzel von Mucor anbetrifft, so ist, wie wir bereits eingangs erwähnten, schon von van Tieghem festgestellt worden, daß eine abgetrennte Hyphe aus sich das Ganze zu bilden vermag. Diese Tatsache wurde zunächst noch einmal experimentell bestätigt. Die weiteren Untersuchungen aber zielten darauf hinaus, zu erfahren, ob auch differenziertere Gebilde, als es die vegetativen Hyphen sind, die Fähigkeit zur Reproduktion eines neuen Organismus besitzen. Infolgedessen wurden von Mucor stolonifer untersucht: die Lufthyphen, die aus dem Myzel als unverzweigte Hyphen in die Luft emporwachsen, die Stolonen und die Rhizoiden, die bei Kontakt mit einem festen Körper an den Stolonen entstehen; der Sporangiumträger und das Sporangium.

Bei Phycomyces nitens beschränkten sich die Versuche auf die Jufthyphen, den Sporangiumträger und das Sporangium.

\section{Mucor stolonifer.}

Obwohl die Ergebnisse van Tieghems in der Hauptsache bestätigt werden konnten, muß doch einiger kleiner Abweichungen halber, 
die sich einstellten, eine mehr wiederholende Darstellung der betreffenden Versuche erfolgen.

Es sei schon hier bemerkt, daß die $\mathrm{zu}$ den Versuchen nötigen Operationen möglichst steril ausgeführt wurden.

Um das vegetative Myzel von Mucor stolonifer zu untersuchen, brachte ich eine Einzelspore in einen Nährtropfen auf Deckgläschen und ließ sie in einer feuchten Kammer (feuchter Papprahmen) auskeimen. Sobald sich ein verzweigtes Myzel gebildet hatte, wandte ich das Deckgläschen um und suchte das Objekt mit Hilfe zweier steriler Nadeln zu zerreißen. Eine nachträgliche Beobachtung zeigte fast stets, daß einige Hyphen verletzt oder vollkommen vom übrigen Myzel abgetrennt waren.

Betrachten wir zunächst eine Wundstelle des Myzels! Ist eine Hyphe nur gedrückt oder gequetscht worden, so ist der Ort der Verletzung daran zu erkennen, daß sich das Plasma an der betreffenden Stelle dunkler gefärbt hat: es stirbt bald ab. War die Verwundung aber so stark, daß eine kleine Öffnung im Zellschlauche entstand, so fließt ein Teil des Plasmas aus, nimmt ebenfalls bald eine dunklere Färbung an und geht zugrunde. In beiden Fällen verläuft die nun folgende Reaktion in gleicher Weise: Auch in dem unmittelbar an die Wunde angrenzenden, intakt gebliebenen Teile der Hyphe färbt sich das Plasma dunkler und stirbt ab. Erst in einiger Entfernung von der Wunde erscheint es wieder hell. Die Grenze zwischen der helleren und dunkleren Partie des Plasmas, zunächst noch etwas undeutlich, tritt nach wenigen Minuten sehr scharf hervor. Es ist die Stelle, wo eine Abschlußmembran gebildet wird, die sich konvex nach der Wunde vorwölbt. Der ganze Vorgang von der Verletzung bis zum Verschluß durch diese Membran nimmt nur kurze Zeit in Anspruch. Was den ersten Teil der Wundreaktion, ehe die Abschlußmembran gebildet wird, anbetrifft, so beobachtete $\mathrm{Ch}$. Ternetz eine ähnliche Erscheinung bei Ascobolus careus, insofern bei diesem Pilze nicht nur die verletzte Zelle, sondern auch die an diese angrenzenden absterben ${ }^{1}$ ).

Dadurch daß nun bei Mucor stolonifer die Bildung einer Vernarbungsmembran auf beiden Seiten der Wunde vor sich geht, wird die verletzte Stelle vollständig isoliert. Damit ist aber das Myzel, wenn es nur an einer Stelle verwundet wurde, in zwei Teile zerlegt worden, die entweder beide größere Komplexe darstellen oder von denen der eine nur durch ein Hyphenende repräsentiert werden kann. In beiden

1) Ch. Ternetz, Jahrb. f. wiss. Bot., Bd. XXXV, pag. 282. 
Fällen lebt jedes der beiden Teilstücke selbständig weiter. Ein auf diese Weise isoliertes Hyphenende fährt fort, mit seiner unverletzten Spitze weiter zu wachsen, es treibt Seitenzweige und bildet schließlich Fruktifikationsorgane, reproduziert also einen ganzen Organismus.

Wird eine Hyphe gleich von Anfang an vollständig vom Myzel getrennt, so spielen sich ganz dieselben Vorgänge ab.

Endlich kann es noch vorkommen, daß Hyphenstücke aus dem Myzel getrennt werden, die auf zwei Seiten verletzt sind. Auch diese bilden dann nach den beiden Wunden hin Abschlußmembranen in der Art, wie oben dargelegt wurde; vorausgesetzt bleibt dabei, daß derartige Teilstücke nicht unter eine gewisse Größe herabsinken.

Soweit stimmen meine Versuche mit denen van Tieghems überein. Nun behauptet dieser Autor noch, daß die verletzte Hyphe ,im allgemeinen“ einen Seitenzweig unter der Wundmembran hervortreibe ${ }^{1}$ ). Das könnte den Glauben erwecken, als ob hier ein korrelativer Zusammenhang bestände zwischen der Verwundung einerseits und der Auszweigung andrerseits, eine Erscheinung, die an manchen Algen festgestellt worden ist aber von mir, so oft ich auch den Versuch wiederholt habe, nicht beobachtet werden konnte. Die Seitenzweige entstanden regellos in einiger Entfernung von der Abschlußmembran.

Es fragt sich nun, ob diese Abschlufmembran selber wachstumsfähig ist, ob sie also die fortwachsende Spitze ersetzen kann. Van Tieghem hat „selten“ gesehen, dafs „die Vernarbungsscheidewand sich selbst weiter entwickelt und daß sich so die Hyphe in ihrer ersten Richtung quer durch den toten Teil verlängert“2). Ich selbst habe eine derartige wachsende Abschlußmembran nicht beobachten können, was nicht ausschließt, daß sie doch die Fähigkeit dazu besitzt, die unter Umständen dann realisiert wird, wenn man die Bildung von Seitenhyphen verhindert.

Von den differenzierten Gebilden untersuchte ich zunächst die Lufthyphen. Diese Lufthyphen repräsentieren im Gegensatz ${ }^{\circ} \mathrm{zu}$ den geschlängelten Hyphen des vegetativen Myzels geradlinig begrenzte Zylinder, die sich außerdem durch ihre Breite und ihre dunklere Färbung auszeichnen. Um sie bequem erlangen zu können, züchtete ich den Pilz auf Brot: Die Lufthyphen schnitt ich mit einer sterilen Scheere $a b$ und brachte sie in einen Hängetropfen oben beschriebener Art. Die durch den Schnitt entstandene Wunde wurde in derselben Weise von dem übrigen Teile der Hyphe durch eine Vernarbungsmem-

1) van Tieghem, l. c. pag. 20.

2) Ders., l. c. pag. 20. 
bran abgeschlossen, wie dies auch an der verletzten vegetativen Hyphe geschieht. An diesen isolierten Lufthyphen erscheinen nun nach der Vernarbung der Wunde starke Seitenzweige (Fig. 1), die sich sofort nach Austritt aus der Mutterhyphe in zwei oder drei Äste teilen und so dieselbe Breite wie die Hyphen des vegetativen Myzels erlangen. Auch hier erfolgt die Anlage der Seitenzweige in bedeutender Entfernung von der Abschlußmembran. Ein Wachstum der unverletzt gebliebenen Spitze, durch das normal die Lufthyphe verlängert wird, findet innerhalb der Nährlösung nicht statt.

Ich wandte mich nun zur Untersuchung des Sporangiumträgers und des Sporangiums. Über die Reaktion des Sporangiumträgers liegen sowohl Tatsachen als Vermutungen vor. „Man wird sich“, meint Goebel, „nicht wundern können, wenn z. B. bei Entfernung eines jungen Mucor-

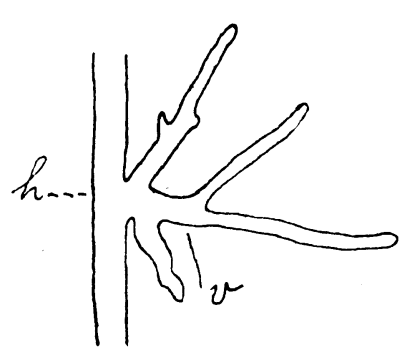

Fig. 1. Lufthyphe $(h)$ von Mucor stolonifer, in Nährlösung untergetaucht, vegetativ aussprossend

(v). Vergr. 250.

\section{Die 2 . meinen Versuchen verwandten Sporangientrager}

Die zu meinen Versuchen verwandten Sporangienträger schnitt ich von ihren Stolonen ab, ließ sie aber in Verbindung mit dem Sporangium. Ich untersuchte also gleichzeitig Sporangiumträger und Sporangium. Die Objekte wurden dann in einen Nährtropfen übertragen, der in üblicher Weise in einer feuchten Kammer gehalten wurde.

Diẹ sofortige Kontrolle der Sporangien und ihrer Träger gab Aufschluß darüber, daß die Untersuchungsobjekte verschieden weit entwickelt waren, und zwar ließen sich im allgemeinen drei Stadien fortgeschrittener Differenzierung unterscheiden: Bei den jüngsten war noch keine Trennungswand zwischen Träger und Sporangium gebildet, das Plasma beider Teile stand infolgedessen in offener Verbindung. Bei älteren Objekten war bereits eine den Stiel und das Sporangium trennende pag. 145 .

1) Goebel, Morphologische und biologische Bemerkungen. Flora 1903,

2) Schröter, Über Protoplasmaströmung bei Mukorineen. Flora, 95. Bd. (1905), pag. 1-30. 
Kolumella entstanden. Auf diesem Stadium bietet das Innere des Sporangiums entweder das Aussehen einer homogenen Masse, oder es sind bereits die ersten Anzeichen einer Zerklüftung des Plasmas in Sporen bemerkbar. Weitere Entwicklungsstadien wurden nicht benutzt, denn schon dann, wenn die Entwicklung des Sporangiums soweit vorgeschritten war, daß sich das Plasma eben in Sporen zu differenzieren begann, hielt die Überführung in Nährlösung die vollständige Ausbildung der Sporen nicht auf. Die Sporen reiften und trieben innerhalb des Sporangiums Hyphen. Dagegen gingen die jüngsten Objekte, die noch keine Kolumella besaßen, innerhalb der Nährlösung zugrunde.

Positive Resultate erzielte ich nur mit den Sporangien, die von ihrem Träger durch eine Kolumella getrennt waren, deren Inneres aber noch als eine homogene Masse erschien. Trotz der zahlreichen Versuche war die Zahl der Resultate sehr gering; denn ich konnte nur zweimal einen Erfolg verzeichnen. Der Verlauf der Reaktion war in beiden Fällen übereinstimmend. Die untergetauchten Sporangien ließen Hyphen auswachsen, die sich in die Höhe richteten, sich über die Oberfläche des Nährtropfens erheben, wo sie an ihren Enden zu kleineren Sporangien anschwollen. Das Bestreben, über die Oberfläche herauszuwachsen, tritt besonders deutlich hervor, wenn auch die Seite des Sporangiums Hyphen treibt, die der Oberfläche des Nährtropfens abgewandt ist: Die Hyphen wachsen erst ein Stück um das Sporangium herum und dann, sobald es ihnen möglich ist, direkt aus dem Substrat heraus. In dem einen Falle waren auf diese Weise sieben neue Sporangien in einer Zeit von 20 Std. entstanden. Das untergetauchte Sporangium selbst war entleert, weil seine Inhaltsstoffe zum Aufbau der Neubildungen gebraucht worden waren.

Bei den vorgehend beschriebenen Versuchen beobachtete ich gleichzeitig den Sporangienträger, der, wie schon gesagt, mit dem Sporangium verbunden blieb. Zunächst zeigte die sofortige Beobachtung nach dem Abtrennen, daß aus der Wunde wenig oder gar kein Plasma ausfloß. Das war auch dann der Fall, wenn die Verbindung zwischen Träger und Sporangium noch offen war. Weiterhin wurde keine Vernarbungsmembran gebildet, wie sie an den Luft- und vegetativen Hyphen entsteht, ebensowenig traten seitliche Aussprossungen auf. Die Reaktion, die Goebel bei dieser Versuchsanordnung vermutet, daß nämlich vegetative Hyphen entstehen, blieb also unter den von mir angewandten Bedingungen aus.

Endlich untersuchte ich auch die Stolonen und Rhizoiden von Mucor stolonifer. Ich verwandte die Stolonen verschiedenen Alters zu 
den Versuchen: Solche, die eben erst einen festen Gegenstand erreicht hatten; solche, an denen die ersten Rhizoiden gebildet waren, und solche, die sich eben anschickten, eine Fruchthyphe entstehen zu lassen. Teils wurden sie vollständig in den Nährtropfen untergetaucht, teils schwammen sie darauf. Ich konnte aber weder die Bildung einer $\mathrm{Ab}$ schlußmembran, noch die Anlage von Seitenzweigen beobachten. Auch die Rhizoiden, die untergetaucht waren, zeigten keinerlei Wachstumstätigkeit. Damit bestätigt sich die Behauptung de Barys, wonach diese Organe nicht reproduzieren, wenn man sie in Nährlösung bringt ${ }^{1}$ ).

2. Phycomyces nitens.

Von Phycomyces nitens untersuchte ich die Lufthyphen, den Sporangiumträger und das Sporangium. Um diese Organe bequem erlangen zu können, kultivierte ich den Pilz auf Brot.

Die Lufthyphen sind wie bei Mucor stolonifer geradlinig begrenzte Zellschläuche. Vor den Lufthyphen von Mucor zeichnen sie sich aber durch ihre bedeutendere Breite und durch ihre dunklere Färbung aus. Ich schnitt sie mit einer sterilen Schere vom Substrat ab, übertrug sie in einen Nährtrōpfen und beobachtete die Wunde, die von Nährflüssigkeit umgeben war. Während bei den Lufthyphen von Mucor stolonifer die einzelnen Phasen der Wundreaktion bis zur Bildung der Vernarbungsmembran mikroskopisch genau verfolgt werden konnten, entziehen sich bei Phycomyces die zunächst auf die Verwundung einsetzenden Vorgänge einer derartigen Betrachtung, weil die dunkel gefärbte Membran einen Einblick in die Tätigkeit des Plasmas verhindert. Der bloß mikroskopischen Beobachtung zeigte das Objekt auch nach Stunden keine Veränderung.

Erst nach einem Zeitraume von 16-20 Stunden bot sich ein anderes Bild dar, das sich aber wesentlich von dem unterscheidet, das an den Lufthyphen von Mucor stolonifer beobachtet wurde. Aus der Lufthyphe von Phycomyces nitens sind nämlich keine seitlichen Hyphen hervorgewachsen, sondern ihr unverletztes Plasma hat sich nach Abschluß mit einer Membran durch den verwundeten Teil hindurch verlängert, indem es zahlreiche Hyphen bildete, die zunächst noch von dem verletzten Zellschlauche wie von einem Zylinder umgeben werden, nach ihrem Austritt aus dieser Hülle aber nach allen Seiten auseinandergehen. Die Lufthyphen von Phycomyces reagieren also ähnlich, wie es van Tieghem in einzelnen Fällen von den vegetativen Hyphen von Mucor angibt.

1) de Bary, Morphologie und Biologie der Pilze, 1884, pag. 48. 
Es galt nun festzustellen, in welcher Weise diese Prolifikationen gebildet werden. Eine Aufhellung mit Kalilauge ließ erkennen, daß die Neubildungen in den meisten Fällen ganz unvermittelt in den unverletzten Teil der Hyphe übergehen. Die Art und Weise, wie die ursprüngliche Vernarbungsmembran angelegt wird, sollte die Untersuchung von Lufthyphen kurz nach ihrer Verwundung zeigen.

$\mathrm{Zu}$ diesem Zwecke wurden solche Hyphen, die noch keine Prolifikationen getrieben hatten, mit Kalilauge aufgehellt. Es wurde beobachtet, daß die Abschlußmembran von Anfang an gewellt ist, sich außerdem ähnlich wie bei Mucor etwas nach außen vorwölbt, aber meist in der Bildung von zahlreichen Prolifikationen aufgeht, so daß eine spätere Untersuchung meist nichts mehr von ihr vorfindet. Figur 2 zeigt ein Objekt, wo ein Teil der Vernarbungsmembran erhalten ist. weil, wie es nur selten geschieht, eine einzige Hyphe gebildet wurde. Wie bei Mucor stolonifer ist der Ort, wo die Vernarbungsmembran angelegt wird, ein bedeutendes Stück von der Wunde entfernt, was darauf hinweist, daß ebenfalls wie bei Mucor

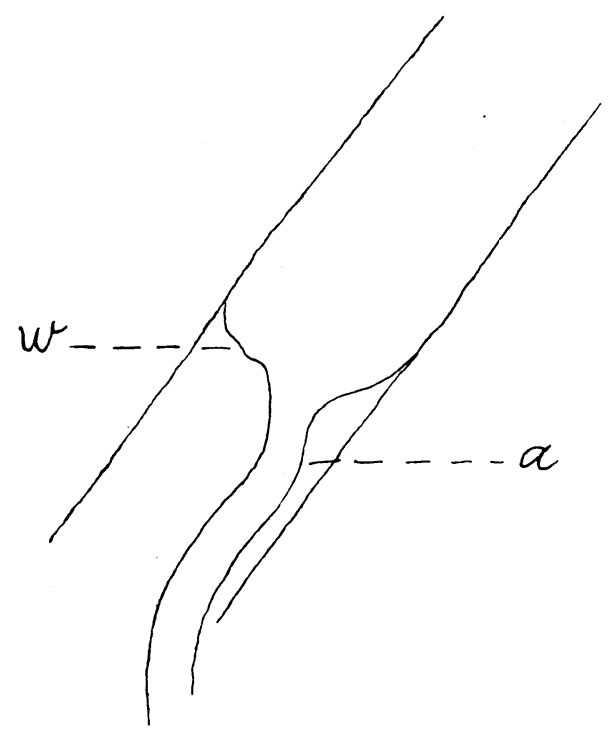

Fig. 2. Hyphenstück von Phycomyces nitens, das bei $w$ eine Abschlußmembran gebildet, die proliferiert $(a)$. Vergr. 350. nicht nur die verwundete Partie, sondern auch ein Teil des angrenzenden, intakt gebliebenen Plasmas abstirbt. Ein analoges Verhalten, wie wir es eben bei Phycomyces kennen lernten, beschreibt Küster an der Alge Anadyomene stellata, wo die langgestreckten Zylinderzellen, nachdem sie den verletzten Protoplasten durch eine Membran geschützt haben, diese Vernarbungsmembran zu englumigen Schläuchen auswachsen lassen ${ }^{1}$ ).

Der Sporangiumträger von Phycomyces nitens wurde wie bei Mucor stolonifer gleichzeitig mit seinem Sporangium untersucht. Er wurde mit einer sterilen Schere vom Substrat abgeschnitten und in

1) E. Küster, Flora 1899, pag. 144 u. 14う. 


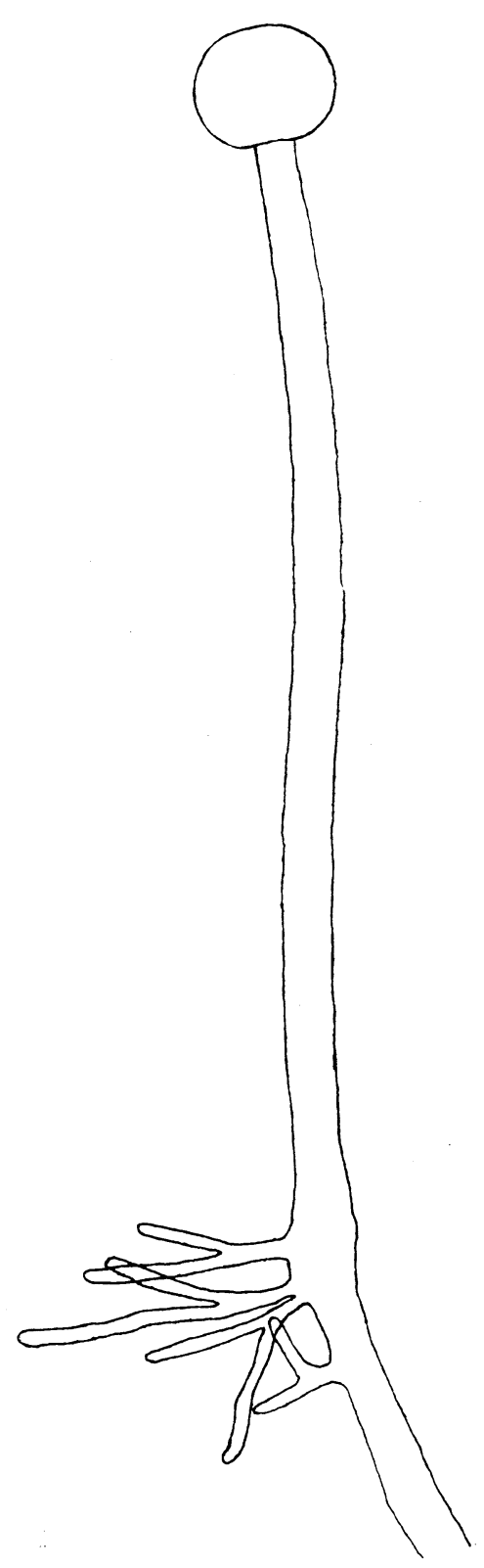

Fig. 3. Sporangiumträger von Phycomyces nitens mit seitlichen Prolifikationen. Vergr. 350. einen Nährtropfen gebracht. In seinem Verhalten glich er vollkommen den Lufthyphen, indem er ebenfalls vegetative Hyphen an der Wundstelle bildete. Dabei bleibt es gleich, wie weit die Wunde von dem Scheitelende, dem Orte, wo der Sporangiumträger normal wächst, entfernt ist; denn der gesamte Protoplast besitzt embryonale Eigenschaft, und wenn das Sporangium abgeschnitten wird, so beginnt der Träger auch an seinem Scheitelende $\mathrm{zu}$ proliferieren.

An dem durch Fig. 3 wiedergegebenen Objekte sind seitlich aus dem Sporangiumträger Hyphen ausgetreten, und sie sind nicht zufällig dort entstanden. Wie die Figur zeigt, beschränken sie sich auf die Stelle, die im Gegensatz zu ihrer Umgebung etwas deformiert erscheint. Das Objekt ist hier bei der Überführung auf das Deckgläschen gedrückt worden, und ich habe es auch des öfteren an Lufthyphen dieses Pilzes experimentell feststellen können, daß eine derartige leichte Quetschung eine lebhafte Hyphenbildung hervorruft, während bei Mucor eine solche Stelle sofort isoliert wird und abstirbt.

Wenn nun noch Fischer konstatierte, daß der Sporangiumträger von Phycomyces nitens unter normalen Außenbedingungen durch eine Verletzung zur Bildung eines Zweiges angeregt wird, der seinerseits wieder zu einem Sporangiumträger wird ${ }^{1}$ ), so bestätigt sich hier die Vermutung, die Goebel vom Sporangiumträger von Mucor ausgesprochen hat (pag. 222). 
Die gleichzeitige Beobachtung der Sporangien ließ dieselben drei Entwicklungsstadien erkennen wie bei den Sporangien von Mucor stolonifer. Entweder war zwischen Sporangiumträger und Sporangium eine Kolumella gebildet oder nicht. Im ersten Falle bestand der Inhalt des Sporangiums aus einer homogenen Masse, oder er begann sich bereits zu Sporen zu zerklüften. Ein Auswachsen, wie es an den Sporangien von Mucor stolonifer konstatiert wurde, blieb aber aus. Die Sporangien verhielten sich vollständig passiv und starben schließlich ab.

\section{Zusammenfassung.}

Die Untersuchungen von Mucor stolonifer und Phycomyces nitens ergaben folgende Resultate:

Mucor stolonifer schließt den verletzten Teil einer vegetativen Hyphe durch eine Vernarbungsmembran von dem lebendigen Plasmakörper ab. Diese Vernarbungsmembran hat nur deckende und schützende Eigenschaft.

Es ist nicht zu erkennen, daß die Verwündung in irgend einer Art und Weise die Produktion der Seitenzweige beeinflußt. Die Seitenzweige entstehen wie unter normalen Verhältnissen.

In derselben Weise wie an den vegetativen Hyphen entsteht auch an den abgetrennten und untergetauchten Lufthyphen in der Nähe der Wunde eine Abschlußmembran, die den verletzten von dem lebendigen Teile der Hyphe trennt. Außerdem treten an beliebigen Stellen vegetative Seitenhyphen auf.

Bei den Lufthyphen von Phycomyces nitens dagegen findet die durch die Verwundung eingeleitete Tätigkeit in der Bildung der Vernarbungsmembran nicht ihren Abschluß; diese Vernarbungsmembran läßt vielmehr zahlreiche Prolifikationen hervorgehen, die in den abgestorbenen Teil der ursprünglichen Hyphe hineinwachsen.

Ebenso entstehen an den Stellen der Lufthyphen von Phycomyces nitens, die leicht gedrückt worden sind, zahlreiche Seitenzweige; bei Mucor stolonifer sterben derartige Partien, auch wenn die Verletzung nur gering war, bald ab und werden dann durch Abschlußmembranen isoliert. Phycomyces nitens scheint demnach bei Verletzungen reaktionsfähiger zu sein als Mucor stolonifer.

Der Sporangiumträger von Mucor stolonifer stirbt im Nährtropfen ab, der von Phycomyces nitens verhält sich genau wie eine Lufthyphe, indem er nach der Wunde hin eine poliferierende Abschlußmembran bildet und unter Umständen auch Seitenzweige treiben kann. 
Das in Nährlösung untergetauchte Sporangium von Mucor stolonifer reproduziert in einzelnen Fällen unter der Bedingung, daß eine Kolumella vorhanden ist und daß die Differenzierung der Sporen noch nicht begonnen hat, Hyphen, die aus der Flüssigkeit hervorwachsen und durch Bildung von Sporangien zu Sporangienträgern werden. An dem Sporangium von Phycomyces nitens dagegen konnten keinerlei Reaktionen konstatiert werden.

Die Versuche mit den Stolonen und Rhizoiden von Mucor stolonifer verliefen resultatlos, insofern die Bildung von Abschlußmembranen von seiten des unverletzten Protoplasten, wie auch die Anlage von Prolifikationen unter den angewandten Versuchsbedingungen ausblieb.

3. Penicillium glaucum und Aspergillus niger.

Bei diesen Pilzen treten uns in zahlreiche Zellen gegliederte Fälen entgegen, wodurch eine stärkere Differenzierung erreicht wird. Zwar unterscheiden wir zunächst ähnlich wie bei den Mucorineen vegetatives Myzel, Lufthyphen und die Organe, die im Zusammenhange mit dem Fortpflanzungsprozesse auftreten, hier die Konidienträger. Vegetatives Myzel und Lufthyphen sind aber weiter differenziert, insofern sie sich aus Scheitel- und Gliederzellen zusammensetzen. Diese Unterscheidung setzt sich auch funktionell fort: das Längenwachstum und die Anlage neuer Gliederzellen beschränkt sich auf die Scheitelzelle, an der außerdem, etwas von der Spitze entfernt, regellos neue Seitenhyphen entstehen. Ein nachträgliches Längenwachstum der einmal angelegten Gliederzellen unterbleibt ${ }^{1}$ ). Dafür treiben aber sehr viele von ihnen gerade so wie die Scheitelzelle Seitenzweige. In der Anlage dieser Seitenzweige zeigt sich, soweit die Gliederzellen von Penicillium glaucum in Betracht kommen, eine gewisse Regelmäßigkeit, indem der Ort der Entstehung in der Regel in die Nähe der oberen, also der Spitze zugekehrten Scheidewand gelegt ist ${ }^{2}$ ).

Es erhebt sich nun die Frage, ob die einzelnen Zellen, die den Organismus dieser beiden Pilze aufbauen, nach dem Abtrennen das Ganze zu reproduzieren vermögen, und ob, falls sie es können, eine Veränderung des ihnen innerhalb des Verbandes aufgeprägten Charakters eintritt.

Die nachfolgenden Untersuchungen sind zunächst an Penicillium glaucum vorgenommen worden.

1) Brefeld, l. c. Heft II, pag. 27 u. 28.

2) Ders., l. c. Heft II, pag. 28. 
Einzelne isolierte Zellen jeder Art und Hyphenstücke, die aus zwei oder mehreren Zellen bestehen, erhält man, wenn man ein Myzelflöckchen aus dem Kulturgefäße auf ein sterilisiertes Deckgläschen überträgt und es hier mit Hilfe von sterilisierten Nadeln mehrfach zerreißt. Nachdem die dadurch entstandenen kleineren Flöckchen entfernt worden sind, setzt man in üblicher Weise mit einem Papprahmen eine feuchte Kammer zusammen und betrachtet dann das Deckgläschen unter dem Mikroskop.

Beobachten wir eine einzelne auf diese Weise isolierte Scheitelzelle! Sie setzt ihr Längenwachstum fort, bildet Querwände, legt also Gliederzellen an und treibt ebenso regellos in einiger Entfernung vom Scheitel Seitenzweige wie im normalen Verbande. Ihr ganzes Verhalten deutet nicht daraufhin, daß durch das $\mathrm{Ab}$ trennen vom Mutterorganismus eine Beeinflussung ihrer Tätigkeit eingetreten ist. Sie erinnert an ein isoliertes Hyphenende von Mucor stolonifer, das, nachdem mit der Bildung einer Abschlußmembran die Wundreaktion vollendet ist, so weiter wächst, als ob es noch mit dem übrigen

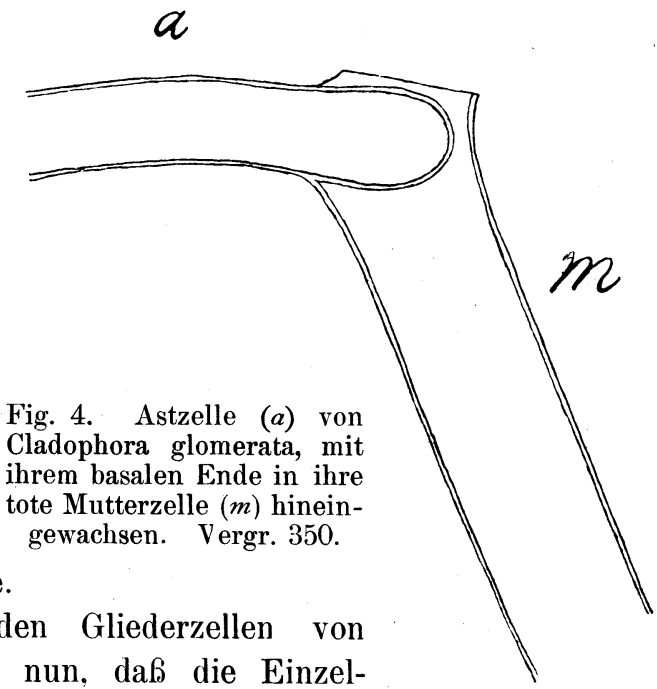
Myzel in Verbindung stände.

Die Versuche mit den Gliederzellen von Penicillium glaucum lehren nun, daß die Einzelzelle mit der Trennung aus der Hyphe eine größere Selbständigkeit gewinnt. Während nämlich die Gliederzellen im normalen Verbande so determiniert werden, daß sie meist nur in der Nähe der oberen Scheidewand Seitenhyphen treiben, wird mit der Isolierung einer Gliederzelle diese Determination aufgehoben, indem die Zelle auch in der Nähe der unteren Scheidewand Seitenzweige hervorgehen läßt. Die Zellen aber, die innerhalb der Hyphe nicht zum Auswachsen bestimmt waren, beweisen durch ihr Aussprossen nach dem Abtrennen, daß trotz der ihnen im Verbande aufgeprägten Ruhe die Wachstumsfähigkeit in ihnen schlummerte. Längenwachstum in der Weise, daß durch die sich vorwölbende Querwand eine fortwachsende Spitze geschaffen wird, tritt aber nicht ein. Auch wenn an einer Hyphe 
die Scheitelzelle entfernt wird, vermag die nachfolgende Gliederzelle nicht das Spitzenwachstum zu übernehmen, wie es z. B. bei manchen Fadenalgen der Fall ist. Fig. 4 gibt eine Astzelle von Cladophora glomerata wieder, deren basale Querwand sich vorgewölbt hat und in die abgestorbene Mutterzelle hineingewachsen ist.

Ich schritt nun zur Untersuchung der Lufthyphen. Lufthyphen erhält man in groß̣er Anzahl, wenn man mit einer sterilen Schere über ein Myzelflöckchen hinschneidet. Morphologisch sind sie vom vegetativen Myzel kaum zu unterscheiden. Auch in ihrer reproduktiven Tätigkeit tritt nichts auf, was nicht schon von den vegetativen Hyphen bekannt wäre. Die einzelnen Zellen der Lufthyphen vermögen sofort in Nährflüssigkeit weiterzuwachsen, indem sie Seitenzweige aussprossen lassen; auf diese Weise vermag eine jede Zelle einen vollständigen Organismus zII reproduzieren.

In derselben Weise verliefen die Versuche mit den Zellen des vegetativen Myzels und der Lufthyphen von Aspergillus niger, insofern sich ebenfalls jede abgetrennte Zelle als fähig erwies, das Ganze zu reproduzieren.

Um die isolierten Konidienträger von Penicillium glaucum und von Aspergillus niger zu erhalten, braucht man nur ein fruktifizierendes Myzel zu zerzupfen, und man wird stets einige Konidienträger, losgetrennt vom Myzel, finden.

Die Konidienträger von Penicillium glaucum setzen sich aus einzelnen Zellen zusammen, die sich weniger durch ihren Habitus als dadurch auszeichnen, daß die Anlage der Aussprossungen streng an den scheitelwärts gelegenen Teil der Zelle gebunden ist. Ein neues Glied wächst immer nur unter der oberen Scheidewand hervor.

Mit der Isolierung der Zellen dieses Konidienträgers geht wie bei den Zellen der vegetativen und Lufthyphen die in der Anlage der Aussprossungen zutage tretende Polarität verloren, insofern auch bei ihnen, sobald sie sich in Nährlösung befinden, die Seitenzweige sowohl unter der oberen, wie auch über der unteren Scheidewand hervorbrechen. In Fig. 5 ist eine derartig proliferierende Konidienträgerzelle dargestellt. Sie trägt noch zwei tote Zellen $(a \mathrm{u} . b)$, und zwar hatte die eine dieser Zellen $(b)$ bereits zwei Konidien $(c)$ abgeschnürt.

Tatsache ist also, daß auch Konidienträgerzellen von Penicillium glaucum in Nährlösung zu vegetierendem Myzel aussprossen.

Eine höhere Ausbildung hat der Konidienträger von Aspergillus niger erfahren. Er stellt nur eine einzige, an ihrem Ende kugelig angeschwollene Zelle dar. Schon in der Einleitung wurde erwähnt, daß 
nach Klebs der Konidienträger von Eurotium herbariorum Hyphen treibt, wenn er mit seinem Myzel in Nährlösung untergetaucht wird. Der isolierte Konidienträger von Aspergillus niger verhielt sich in Nährlösung genau so; dabei war es, wie auch bei Eurotium herbariorum, gleichgiltig, ob die Anlagen der Konidien bereits gebildet waren oder nicht. In der Nähe des Scheitels gingen in der Regel 2-4 vegetative Hyphen hervor. In diesem Konidienträger tritt uns damit ein Organ entgegen. das auch isoliert den Gegensatz zwischen Basis und Scheitel aufrecht erhält.

Beobachtungen über die Regenerationsfähigkeit des nackten Protoplasten hat Brefeld an der Scheitelzelle von Penicillium glaucum gemacht ${ }^{1}$ ). Durch heftige Erschütterungen brachte er die Membran der Scheitelzelle zum Platzen, so daß ein Teil des Plasmas wurstartig hervorquoll. Um diesen nackten Protoplasten bildete sich von neuem eine Membran, wodurch die Wunde wieder verheilt wurde. $\mathrm{Ob}$ diese regenerierten Partien auch wachstumsfähig waren und Seitenzweige trieben, erfahren wir nicht. Immerhin bleibt ein derartiges Verhalten nicht ausgeschlossen. Ich selbst habe es nicht beobachten können.

\section{Zusammenfassung.}

Bei Penicillium glaucum und Aspergillus niger ist eine jede einzelne Zelle befähigt, die embryonale Tätigkeit aufzunehmen. Wie

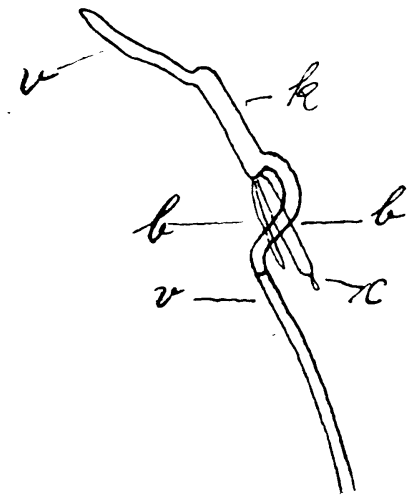

Fig. 5. Konidienträgerzelle $(h)$ von Penicillium glaucum, in Nährlösung untergetaucht, regetative Hyphen (v) treibend. $b=$ zwei tragende Zellen, die abgestorben sind; $c$ abgestorbene Konidienanlage. Vergr. 350. weit ohne Einbuße der embryonalen Eigenschaften die formative Änderung einer Zelle gehen darf, lehren z. B. die Versuche mit dem Konidienträger von Aspergillus niger. Ein solches Verhalten ist natürlich nur bei niederen Organismen möglich, da bei höheren Pflanzen .,die Ausgestaltung von Organen und Geweben besonderer morphologischer und funktioneller Bedeutung eine bestimmte Differenzierung fordert" 2). Bei diesen Organismen, die sich „noch nicht allzuweit vom Begriffe der Zellkolonie entfernen" ${ }^{3}$ ), besitzt jede Zelle noch eine ziemlich große Selbständigkeit, die dann zutage tritt, wenn die durch den Verband bedingten Korrelationen aufgehoben werden.

1) Brefeld, l. c. Heft II. pag. 29.

2) Pfeffer, l. c. Bd. II, pag. 4 .

3) Tobler, Ber. d. bot. Gesellsch., Bd. XXI, pag. 299. 


\section{Versuche mit höheren Pílzen.}

Als Versuchsobjekte verwandte ich in erster Linie zwei Pilze, an denen bereits ausgedehntere Untersuchungen gemacht worden sind: Coprinus ephemerus und Agaricus campestris. Damit waren mir zwei Vertreter der Pilze gegeben, deren Fruchtkörper stets scharf in Hut und Stiel gegliedert ist. Weitere Versuche stellte ich mit Formen der Gattung Xylaria an, und zwar standen mir zwei Objekte zur. Verfügung: Xylaria hypoxylon und Xylaria arbuscula, eine Art, die man oft an den Kübeln tropischer Pflanzen in Gewächshäusern findet. - Von den Pilzen, deren Fruchtkörper in der Regel weniger differenziert ist und wie sie zu einem großen Teil den Polyporeen angehören, benutzte ich Polyporus versicolor, Polyporus candicinus, Polyporus Braunii und Daedalea unicolor. Außerdem untersuchte ich noch das Sklerotium von Claviceps purpurea.

Was über die Reproduktions- und Regenerationsfähigkeit dieser und anderer höherer Pilze bereits bekannt ist, habe ich in der Einleitung zusammengestellt. Gelegentlich werde ich an gegebener Stelle noch ausführlicher darauf zurückkommen.

\section{Coprinus ephemerus.}

$\mathrm{Zu}$ meinen Untersuchungen Coprinus ephemerus heranzuziehen, bewog mich der Umstand, daß Brefeld nur eine kurze Mitteilung von der Reaktionsfähigkeit dieses Pilzes macht. Er beschränkt sich nämlich auf folgende Angabe: „Die Fruchtkörper sprossen vor ihrer Sporenreife je nach Umständen zu neuen Fruchtkörpern oder Myzelien aus, und zwar sogleich in jeder Zelle und in jedem Stadium der Entwicklung“" 1). Außerdem läßt er die Frage offen, ob der Fruchtkörper von Coprinus ephemerus ebenso den weggeschnittenen Hut zu regenerieren vermag, wie es bei Coprinus stercorarius konstatiert wurde.

Um den Fruchtkörper von Coprinus ephemerus zu erhalten, bedeckt man frischen Pferdemist mit einer Glasglocke. Man hat nur dafür zu sorgen, daß das Substrat in mäßiger Feuchtigkeit erhalten wird und genügendes Licht bekommt, und nach ungefähr 10 Tagen erscheinen dann die ersten Fruchtkörper von Coprinus ephemerus.

Es fällt nun nicht schwer, eine Reinkultur von diesem Pilze herzustellen. Man sterilisiert Pferdemist, der sich in Kristallisierschalen befindet an drei aufeinanderfolgenden Tagen, jedesmal ungefähr 2 Stunden, und verfährt dann in folgender Weise: man löst einen Fruchtkörper, von dem man annimmt, daß er noch im Laufe der folgenden Nacht

1) Brefeld, l. c. Heft III, pag. 114. 
fruktifizieren wird, von seinem Substrat und befestigt ihn über sterilisiertem Papier, das sich in einer ebenfalls keimfreien Kristallisierschale befindet. Am nächsten Morgèn hat der Fruchtkörper zahlreiche Sporen ausgestreut, und man kann nun mit diesen Sporen den sterilisierten Mist impfen. Auf diesem Substrat kommen dann nach etwa 3 Wochen die ersten jungen Fruchtkörper hervor.

Teilstücke von Fruchtkörpern, die bereits Hut und Stiel differenziert hatten, untersuchte ich in der Weise, daß ich eine Nahrungszufuhr von außen möglichst $\mathrm{zu}$ verhindern suchte. $\mathrm{Zu}$ diesem $\mathrm{Zwecke}$ hob ich Fruchtkörper von ihrem Nährsubstrat ab und halbierte sie auf einem sterilisierten Objektträger durch einen Längschnitt, so daß jedes Teilstück aus einem halbierten Hute und einem halbierten Stiele bestand. Ich übertrug diese Teilstücke auf feuchtes Fließpapier, das in einer Kristallisierschale ausgebreitet war. Kristallisierschale mit Fließpapier hatte ich vorher sterilisiert. Nach einigen Tagen entstanden an den Teilstücken Wucherungen von seiten der Hyphen. Nach meinen Beobachtungen traten an einem Teilstück nie mehr als zwei solcher Hyphenwucherungen auf, in der Regel war es nur eine. Wie eine Betrachtung unter der Lupe lehrte, wurden diese Wucherungen von einem lockeren Hyphengeflecht gebildet. Der Ort ihrer Entstehung war ganz beliebig gewählt. Sie traten sowohl am Stiele, als auch am Hute auf, und zwar ebenso oft auf der Schnittfläche, wie auf den unverletzten Partieen; sogar an den Lamellen waren sie hin und wieder zu konstatieren.

Die Hyphenwucherungen verdichteten sich allmählich und ließen ganz deutlich Stiel und Hut hervortreten. Eine Entwicklung bis zur Sporenreife konnte jedoch in keinem Falle beobachtet werden.

Bei anderen Versuchen verfuhr ich in der Weise, daß ich zunächst ebenfalls den Fruchtkörper vom Substrat abhob, dann aber den Hut vom Stiel trennte und so beide Teile isoliert auf Fließpapier auslegte. Auch stellte ich noch kleinere Teilstücke her, indem ich einmal den Fruchtkörper durch zwei aufeinander senkrecht stehende Längsschnitte in vier Teile zerlegte, ein anderes Mal den isolierten Hut oder Stiel halbierte. Der Erfolg war derselbe, wie er oben beschrieben wurde: An allen diesen Teilstücken traten 1-2 neue Fruchtkörper auf. Wieder waren es sowohl die Oberflächenzellen, als auch die Zellen der Schnittflächen am Hut und am Stiel, von denen die Ersatztätigkeit ausgeführt wurde. Auch Lamellenzellen begannen in einzelnen Fällen auszusprossen, um dann weiterhin einen neuen Fruchtkörper entstehen zu lassen. 
Ein Vergleich mit den entsprechenden Untersuchungen Brefelds an Coprinus stercorarius läßt einige kleine Unterschiede in den Resultaten erkennen.

Zunächst konnte Brefeld fast stets eine größere Anzahl von reproduzierten Fruchtkörpern feststellen. Am inneren Rande eines isolierten Hutes von Coprinus stercorarius entstanden z. B. in einem Falle gegen 70 Sprossungen, ,und jede von ihnen kam soweit in der Entwicklung, daß man Hut, Stiel und Volva deutlich erkennen konnte" ${ }^{1}$ ). Dagegen sah ich bei meinen Versuchen zunächst nur einen neuen Fruchtkörper hervorgehen, in wenigen Fällen waren es zwei, und zwar war das auch der Fall bei den größten Teilstücken, die ich verwandte.

Weiter hebt Brefeld hervor, „daß vorzugsweise die Schnittflächen auswachsen" 2). Dagegen mußte ich feststellen, daß bei Coprinus ephemerus von einer Bevorzugung der Wundstelle nichts $\mathrm{zu}$ merken war; denn die Wucherungen traten ebenso oft an den unverletzten Teilen des Stieles und des Hutes auf wie an deren Schnittflächen.

Was aber Brefeld ais wesentlichstes Ergebnis bei seinen Versuchen an Coprinus stercorarius und Coprinus ephemerus fand, daß nämlich reproduktionsfähige Zellen in allen Teilen des Fruchtkörpers vorhanden sind ${ }^{3}$, wurde bei Coprinus ephemerus noch, einmal bestätigt.

In eine ganz andere Richtung wird die Ersatztätigkeit gelenkt, wenn die Außenbedingungen in der Weise geändert werden, daß das Versuchsobjekt anstatt in eine nahrungsarme Umgebung auf ein nährstoffreiches Substrat gebracht wird. Wie bei den vorhergehenden Versuchen halbierte ich junge Fruchtkörper von Coprinus ephemerus durch Längsschnitte, legte aber jetzt die Teilstücke auf sterilisierten Mist, der sich in gleichfalls sterilisierten Kristallisierschalen befand. Der Erfolg war der, den auch Brefeld an diesem Pilze, wie an Coprinus stercorarius eintreten $\operatorname{sah}^{4}$ ). Schon am nächsten Tage nach der Auslage beginnt die größte Zahl der nach außen gelegenen Zellen des Hutes und des Stieles, und zwar sowohl an der unverletzten Oberfläche, wie an den Schnittflächen zu Myzel auszuwachsen. - Dasselbe gelang auch an kleineren Teilstücken, die ich aus dem Fruchtkörper herstellte.

Durch diese Versuche wird noch einmal bestätigt. was sich schon aus den ersten Versuchen ergab, daß nämlich trotz der hohen Differenzierung des Fruchtkörpers Hut- und Stielzellen die Fähigkeit zum
1) Brefeld, l. c. Heft III, pag. 74.
2) Ders., 1. c. Heft III, pag. 74 .
3) Ders., l. c. Heft III, pag. 74 u. 75 .
4) Ders., l. c. Heft III, pag. $80 \mathrm{ff}$. 
Auswachsen besitzen und zum Ausgangspunkt neuer Organismen werden können.

Es galt nun noch zu entscheiden, ob auch der auf dem Nährsubstrat stehenbleibende Fruchtträger von Coprinus ephemerus den weggeschnittenen Hut zu regenerieren vermag, wie es Brefeld bei Coprinus stercorarius beobachtete. $\mathrm{Zu}$ meinen Versuchen wählte ich verhältnismäßig junge, aber kräftige Objekte aus, denen ich mit einem schnellen Schnitt den Hut abtrennte. Fine Sprossung der durch die Verletzung freigelegten Stielzellen zwecks einer Regeneration des Hutes unterblieb jedoch. Aber auch seitlich, an dem unverletzten Teile des Stieles traten keine Wucherungen auf, wie es Brefeld wahrnahm, wenn er, um eine Regeneration zu verhindern, die Schnittfläche verklebte ${ }^{1}$ ). Dagegen wuchsen in der Nähe der Stielbasis, aber vom Myzel aus eine Anzahl, meist 3-5, kümmerlicher Fruchtkörper hervor. Oft hatte es den Anschein, als ob sie ihre Entstehung aus dem Fruchtträger genommen hätten. Eine genauere Untersuchung ergab aber stets, daß ihre Bildung vom Myzel ausging. Offenbar steht diese Erscheinung in einem korrelativen Zusammenhange mit der Verletzung. Auch an 'dem Sklerotium von Coprinus stercorarius, auf dem bei Brefelds Versuch der seines Hutes beraubte Fruchtträger stehen blieb, keimten nach dem Abschneiden des Hutes Fruchtanlagen aus ${ }^{2}$ ), die von Brefeld unterdrückt wurden. Bei Coprinus ephemerus verhinderte ich ebenfalls die Bildung von Fruchtkörpern in der Nähe der Stielbasis, trotzdem kam keine Regeneration zustande. In den Stielzellen konnte die Ursache nicht liegen, daß die erwartete Reaktion ausblieb; denn deren Fähigkeit, in den embryonalen Zustand zurückzukehren, ist durch die vorhergehenden Versuche erwiesen. Jedenfalls ist es so, daß Korrelationen, die durch die reproduktive Ersatztätigkeit ausgelöst werden, die Regeneration unterdrücken ${ }^{3}$ ). Ähnliche Erscheinungen werden uns im Laufe der Arbeit noch mehrfach entgegentreten.

\section{Agaricus campestris.}

Der mannigfachen Ersatztätigkeiten reproduktiver wie regenerativer Art, die an diesem Pilze bereits bekannt geworden sind, wurde schon in der Einleitung gedacht. Jene Mitteilungen weisen darauf hin, daß in Agaricus campestris ein für unsere Untersuchungen günstiges Objekt gegeben ist. Es lag nahe, auch diesen Pilz zu Versuchen heranzuziehen.

1) Brefeld, l. c. Heft III, pag. 70.

2) Ders., l. c. Heft III, pag. 69.

3) Vergl. hierüber Pf effer, l. c. Bd. II, pag. 208.

Flora 1907. 
Die durch die Versuche mit Coprinus ephemerus gewonnenen Erfahrungen lehren, daß noch in relativ kleinen Teilen eines Fruchtkörpers soviel Nährstoffe enthalten sein können, daß auf Kosten dieser Baustoffe die Bildung neuer, wenn auch kleiner Fruchtkörper stattfinden kann; vorausgesetzt bleibt natürlich, daß dazu befähigte Zellen vorhanden sind. Von diesem Gedanken ging ich bei der ersten Versuchsreihe, die ich anstellte, aus.

Ich verwandte Fruchtkörper, an denen bereits Hut und Stiel differenziert waren. Der Hut war jedoch noch geschlossen oder begann eben, seinen Rand vom Stiel zu lösen. Infolgedessen wurden die Lamellen, die ebenfalls schon ausgebildet waren, noch vom Hutrande verdeckt, so daß sie am unverletzten Objekte nicht zu sehen waren. Die Höhe der untersuchten Fruchtkörper bewegte sich zwischen etwa 1,5 bis $3,5 \mathrm{~cm}$.

Aus diesen Bemerkungen ist zu ersehen, daß die Objekte bereits sehr weit in der Entwicklung vorgeschritten waren. Daß in diesem Stadium wie bei Coprinus ephemerus und Coprinus stercorarius die Fähigkeit der Zellen, in andere Wachstumsrichtung gelenkt zu werden, noch nicht verloren ging, wird aus den Versuchen ersichtlich werden.

Ich verfuhr nun so, daß ich den Hut vom Stiel trennte und beide Teile, Hut und Stiel, in Kristallisierschalen auf feuchtes Fließpapier auslegte. Nach 3-4 Tagen machte sich an den isolierten Stielen der erste Erfolg bemerkbar, indem auf der Schnittfläche ein weißer Überzug erschien, der, wie die mikroskopische Untersuchung ergab, auf eine Sprossung der den Stiel aufbauenden Hyphen zurückzuführen war. Am intensivsten wachsen die Hyphen im Zentrum der Schnittfläche. Nach der Peripherie zu wird das Wachstum immer geringer, bis es schließlich ganz aufhört, so daß der Hyphenschopf eine Fläche bedeckt, die etwa den halben Durchmesser der gesamten Schnittfläche besitzt. Die Deutung dieser Hyphensprossung behalten wir uns vor, bis eine andere Erscheinung, die ebenfalls an dem isolierten Stiele von Agaricus campestris auftrat, beschrieben worden ist.

Unter dem Versuchsmaterial befand sich ein sogenannter Zwilling, d. h. zwei Fruchtkörper waren an der Basis ihrer Stiele miteinander verwachsen; diese Stelle ist in Fig. 6 mit $a$ bezeichnet. Rechts von $a$ befindet sich der eine Stiel, der an seinem Ende $b$, wo der Hut aufsaß, etwas angeschwollen erscheint. Links etwas schräg in die Höhe geht der andere Fruchtträger. In $c$ erkennen wir die Fläche, die durch die Entfernung des Hutes frei gelegt wurde. Der weiße Fleck, der mit $d$ bezeichnet worden ist, ist der Hyphenschopf des Stieles, wie er als all- 
gemein auftretend schon oben dargestellt wurde. An der Peripherie der Schnittfläche ist aber noch ein anderes Gebilde entstanden.

Schon eine flüchtige Betrachtung belehrt uns, daß ein neuer Fruchtkörper reproduziert worden ist. Sehen wir uns aber die Neubildung genauer an, so treten eine Anzahl von Eigentümlichkeiten hervor, die an einem normalen Fruchtkörper nicht zu beobachten sind. Zunächst fallen die unregelmäßig angeordneten Lamellen $(e)$ auf, die auch nicht wie normal in einer horizontalen, sondern in einer vertikalen Ebene angelegt wurden. Außerdem sind sie nur auf der dem reproduzierenden Teilstück abgewandten Seite ausgebildet und liegen von Anfang an frei da. Über den Lamellen sehen wir ein hutartig gewölbtes Gebilde. Was sich unter den Lamellen befindet, könnten wir nach Habitus und Orientierung als Stiel ansprechen. Bemerkenswert ist noch die Art und Weise, wie die Neubildung an dem reproduzierenden Stück befestigt ist. Die Verbindung mit dem Mutterstück hat nicht der stielartige Teil, sondern die hutartig ausgebildete Partie übernommen. Infolgedessen hängt der Stiel, wie wir diesen Teil nennen wollen, ganz frei nach unten. Die Ansatzstelle $(f)$ des neuen Fruchtkörpers weist nur eine Stärke von ca. $2 \mathrm{~mm}$ auf.

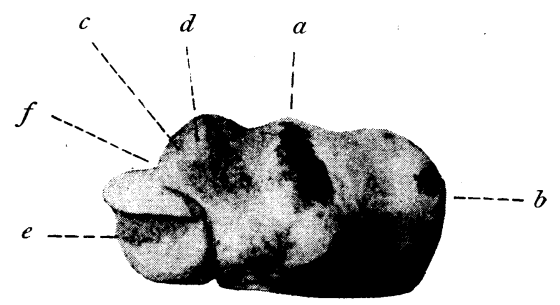

Fig. 6. Stiel von Agaricus campestris, mit reproduziertem Fruchtkörper. Etwas verkleinert.

$\mathrm{Da}$ Teilstücke von Fruchtkörpern die Fähigkeit zur Reproduktion neuer Fruchtkörper besitzen können, wurde bereits durch die Untersuchungen Brefelds an Coprinus festgestellt. Infolgedessen bringt die soeben beschriebene Erscheinung generell nichts neues. Nur in der morphologischen Ausgestaltung des reproduzierten Gebildes tritt ein Unterschied hervor. Denn während an den Teilstücken von Coprinus normale Fruchtkörper angelegt wurden, nahm der neue Fruchtkörper am isolierten Stiele von Agaricus campestris eine Form an, die von der normalen bedeutend abweicht.

Brefeld hat nun eine Anzahl Stiele von Coprinus stercorarius, denen er den Hut weggeschnitten hatte und die dann neue normale Fruchtkörper reproduzierten, durch Zeichnung wiedergegeben ${ }^{1}$ ). Die Betrachtung dieser Figuren läßt eine weitere Übereinstimmung zwischen Coprinus und Agaricus erkennen. Denn fast alle Stiele von Coprinus

1) Brefeld, l. c. Heft III, Tafel III, Erklärung dazu pag. 210. 
stercorarius tragen auf ihrer Schnittfläche einen Hyphenschopf, wie er auch auf der Schnittfläche vieler Stiele von Agaricus campestris entstand und wie ihn auch die Schnittfläche des in Fig. $6 d$ dargestellten Objektes zeigt. Auch bei Coprinus stercorarius sind an dieser Bildung nur die im Zentrum der Schnittfläche gelegenen Zellen beteiligt. Brefeld erwähnt diese Bildung erst in der Erklärung seiner Figuren.

Da wir wissen, daß bei Coprinus stercorarius alle Stielzellen zum Auswachsen befähigt sind, so kann das Aussprossen der mittleren und das Verharren der äußeren im Ruhezustande nur der Ausdruck eines korrelativen Wirkens sein. Tatsächlich ging auch in einigen Fällen aus einem solchen Hyphenschopf ein neuer Fruchtkörper hervor. Brefeld nennt deshalb die nicht zur Entwicklung kommenden Hyphenschöpfe „primäre Fruchtanlagen"1).

Haben wir Grund, die Hyphensprossungen auf der Schnittfäche des Stiels von Agaricus campestris in derselben Weise zu deuten? Da bei Agaricus campestris die Bildung eines Fruchtkörpers aus einem solchen Hyphenschopfe unterblieb, so erhält eine solche Deutung erst dann eine gewisse Berechtigung, wenn wir nachweisen können, daß, wie bei Coprinus stercorarius, alle freigelegten Stielzellen wachstumsfähig sind. Denn dann müßte ebenfalls das Auswachsen eines beschränkten Teils befähigter Zellen als Erfolg eines korrelativen Wirkens aufgefaßt werden, das natürlich keinen anderen Zweck haben kann, als einen neuen Fruchtkörper zu bilḍen. Der Nachweis von der Wachstumsfähigkeit aller Stielzellen soll durch einen anderen Versuch erbracht werden.

Ich schnitt sowohl aus dem Stiel, als auch aus dem Hute sterile Teilstücke heraus und brachte sie in eine mit feuchtem Fließpapier ausgelegte, sterilisierte Kristallisierschale. An diesen Teilstücken entstanden ebenfalls Hyphensprossungen. Sie unterscheiden sich aber wesentlich von denen, die am isolierten Stiele auftraten. Denn während am Stiele nur die Hyphen einer bestimmten Stelle, nämlich der Mitte der Schnittfläche zu sprossen begannen, sind an diesen Teilstücken die wachsenden Hyphen über die ganze Außenfläche verteilt, so daß diese mit einem feinen weißen Überzuge bedeckt erscheint. Diese Tatsache beweist zunächst, daß wohl alle Zellen des Hut- wie des Stielinneren zu wachsen vermögen; auf jeden Fall sind mit dieser Fähigkeit nicht nur die wenigen Zellen ausgestattet, die auf der Schnittfläche des Stieles aussprossen. Da weiter an diesen Teilstücken keine Stelle vor der anderen

1) Brefeld, 1. c. Heft III, pag. 210. 
bevorzugt ist, die Sprossungen vielmehr gleichmäßig über die ganze Oberfläche verteilt sind, so darf man wohl annehmen, daß am isolierten Stiele von Agaricus campestris wie bei Coprinus stercorarius innere Einflüsse vorliegen, die auf Bildung eines neuen Fruchtkörpers auf der Schnittlläche hinzielen, und es bleibt nicht ausgeschlossen, daß man noch einen derartigen Erfolg verzeichnen kann.

Da beide Pilze, Agaricus und Coprinus, bis jetzt so augenfällig übereinstimmten, so mußte die Frage auftauchen, ob es nicht gerade so wie bei Coprinus stercorarius möglich sei, die aussprossenden Zellen, die an isolierten Teilstücken von Agaricus campestris auftreten, in vegetatives Myzel üherzuführen.

Steril aus Hut oder Stiel herauspräparierte Teilstücke wurden deshalb auf sterilisierten Mist gelegt. der sich in sterilisierten Kristallisierschalen befand. Auch an diesen Teilstücken begannen die nach außen gelegenen Zellen in der Weise auszusprossen, wie es oben beschrieben worden ist. Es gelang jedoch nicht, die Teilstücke zur Bildung von Myzel zu bringen, was nach den Gesamterfahrungen anzunehmen berechtigt war.

3. Xylaria arbuscula und Xylaria hypoxylon.

Durch Hennings ist schon eine reproduktionsfähige Form von Xylaria bekannt geworden, und zwar gehört sie der Gattung Kretschmeria an ${ }^{1}$. Wenn die Spitzen der jungen Konidienpilze von Nacktschnecken abgenagt werden, so entwickeln sich auf den zurückbleibenden Stielstümpfen „kopfförmige Fruchtkörper“. Da, wie wir wissen, Xylaria nacheinander zwei Arten von Fortpflanzungsorganen hervorbringt, nämlich zuerst Konidien und darnach in Perithezien die morphologisch höher stehenden Askussporen, so fragt es sich, welche von beiden Arten die reproduzierten Fruchtkörper, die Hennings beobachtete, gebildet haben. Hennings gibt darüber keine Auskunft. Uns war die Entscheidung dieser Frage unmöglich, da uns der Pilz nicht zur Verfügung stand.

Dagegen wurde eine große Anzahl ähnlicher und anderer Operationen mit Xylaria arbuscula angestellt. Xylaria arbuscula ist wie Kretschmeria ein tropischer Pilz, der im Warmhause auf einem Kübel gefunden wurde, an dessen Innenrande er einen ausgedehnten Rasen bildete.

Die durch Schneckenfraß hervorgerufene und von Hennings beobachtete Reproduktion versuchte ich an Xylaria arbuscula in der Weise

1) Hennings, l. c. pag. 140 . 
zu wiederholen, daß ich solchen Objekten, die noch nicht fruktifiziert hatten, die äußersten, weißlich gefärbten Spitzen in einer Länge von durchschnittlich $1 \mathrm{~mm}$ abschnitt. Der verletzte Pilz blieb während des Versuchs auf seinem Substrat stehen. Die nun einsetzende Wachstumstätigkeit brachte ein anderes Gebilde hervor, als es Hennings an Kretschmeria beschreibt.

Schon nach 24 Stunden waren sämtliche durch den Schnitt freigelegten Hyphen ausgesproßt und hatten nach 3-4 Tagen die weggenommenen Spitzen so vollkommen ersetzt, daß auf Längsschnitten der Ort der Verwundung nicht wiedergefunden werden konnte. Es liegt also hier ein Fall echter Regeneration vor.

Bei den weiteren Untersuchungen mit Xylaria arbuscula verfuhr ich nach der Methode, die schon bei Coprinus und Agaricus mit Erfolg angewandt wurde. Ich stellte Teilstücke her, die ich in Kristallisierschalen auf feuchtes Fließpapier auslegte. Eine Variation der in dieser Weise angestellten Versuche war deshalb möglich, weil Xylaria arbuscula in allen Phasen fortschreitender Entwicklung bis und nach der Bildung der Askussporen reaktionsfähig bleibt.

Nach der angedeuteten Methode behandelte ich noch einmal solche Objekte, die sich als fähig erwiesen hatten, die weggeschnittene Spitze zu regenerieren, an denen also noch keine Fortpflanzungsorgane gebildet waren. Die etwa $3 \mathrm{~mm}$ langen, auf feuchtes Fließpapier ausgelegten und durch Querschnitte hergestellten Teilstücke hatten nach Verlauf von 2 Tagen auf beiden Schnittflächen weiße Hyphenschöpfe reproduziert, die eine Länge von 1,0-1,5 mm gewannen. Zwei bis drei Tage später lehrte ein feiner weißer Staub, der bei der geringsten Berührung niederrieselte, sowie die mikroskopische Betrachtung dünner Längsschnitte, daß die hervorgesprossenen Hyphen Konidien abgeschnürt hatten. Gleichzeitig bestätigte das mikroskopische Bild, was schon eine makroskopische Betrachtung vermuten ließ, daß nämlich nur die weißen Markhyphen an der Sprossung beteiligt sind, während die unter dem Mikroskop bräunlich gelb erscheinenden Rindenzellen sich passiv verhalten.

Die Rindenschicht besteht, wie schon de Bary angibt ${ }^{1}$ ), aus toten Zellen, die infolgedessen nicht an der Reproduktion teilnehmen können. Das geht auch aus einem anderen Versuche hervor, zu dem ich aber Objekte nahm, die an ihren Spitzen bereits Konidien trugen. Ich verfuhr so, daß ich die Enden der Teilstücke, die wiederum durch Quer-

1) de Bary, l. c. pag. 60 . 
schnitte hergestellt waren, in einer Bunsenflamme abtötete, um die Schnittflächen am Aussprossen zu verhindern. Etwa 2 Tage nach dieser Operation trat an irgend einer vorher nicht zu erkennenden oder zu bestimmenden Stelle der unverletzten Außenseite des Teilstücks eine Hyphensprossung auf, die sich durch ihre weiße Färbung scharf gegen die schwarze Rinde abhob. Auf dünnen Querschnitten kann man sehen, wie die Sprossungen entstehen. Unter der Rindenschicht findet eine lebhafte Vermehrung der Markhyphen statt, so daß schließlich die Rinde gesprengt und von den wachsenden Hyphen beiseite gedirängt wird.

In vielen Fällen hört aber das Wachstum der Sprossung nach dem Durchbruch durch die Rinde noch nicht auf. Wie bei den entsprechenden Versuchen an Coprinus ephemerus und Agaricus campestris wird vielmehr die Bildung eines neuen Fruchtkörpers angestrebt. Die Sprossung verlängert sich und steht in einem Winkel von $4 \tilde{5}-90^{\circ}$ zweigartig vom Teilstück ab. Gleichzeitig färben sich von der Basis aus die Zellen der Außenseite dunkler, so daß schließlich nur die fortwachsende Spitze die ursprüngliche weiße Färbung bewahrt. Hört das Wachstum endlich auf, so werden von dieser Spitze Konidien abgeschnürt. Die ganze Bildung zeigt jetzt alle Charaktere eines normalen Fruchtkörpers auf der Stufe der Konidienfruktifikation. In einzelnen Fällen übertrifft der neue Fruchtkörper bei weitem die Länge des reproduzierenden Teilstücks; dafür bleibt aber dann, der beschränkten Nahrungszufuhr entsprechend, der Querschnitt sehr schmal.

Die Reproduktion von Fruchtkörpern, wie sie eben konstatiert wurde, wird auch, und das macht diesen Pilz besonders interessant, an älteren Objekten ausgeführt, die bereits die beiden für diesen Pilz so charakteristischen Formen der Fruchtbildung durchlaufen haben. Derartige ältere Objekte, die sich an dem keulenförmig angeschwollenen Stroma kenntlich machen, schneidet man vom Substrat ab und bringt sie auf feuchtes Fließpapier, wo sie nach etwa 2 Tagen auszusprossen beginnen. Die Aussprossungen werden meist unter der schwarzen Rindenschicht des Fruchtträgers, die sie dann durchbrechen, angelegt; hin und wieder entstehen sie auch auf der Schnittfläche des. Fruchtträgers. In allen Fällen stellen sie die Anlagen neuer Fruchtkörper dar, deren vollstänständige Ausbildung sich nach einem Verlauf von weiteren 3 Tagen vollzogen hat. So zeigt Fig. 7 einen ausgelegten Fruchtkörper, an dessen Stiel fünf neue Fruchtkörper produziert wurden, und zwar ist einer dieser Fruchtkörper auf der Schnittfläche des Stiels $(h)$ entstanden. Die Spitzen der jungen Fruchtkörper schnüren Konidien ab, die bei der geringsten Erschütterung in großen Mengen zu Boden fallen. 
Ich veränderte nun den Versuch in der Weise, daß ich das Stroma vom Fruchtträger trennte und so beide Teile isoliert betrachten konnte. Der Fruchtträger produzierte wieder eine Anzahl neuer Fruchtkörper, während das Stroma keinerlei Wachstumstätigkeit erkennen ließ, anscheinend sterben seine Zellen sofort nach der Bildung der Sporen ab.

Überblicken wir noch einmal die mit Xylaria arbuscula unternommenen Versuche, so gelangen wir zu folgenden Ergebnissen:

Wie bei Coprinus ephemerus konnte nachgewiesen werden, daß alle lebenden Zellen des Fruchtkörpers von Xylaria arbuscula die Fähigkeit zur Reproduktion besitzen.

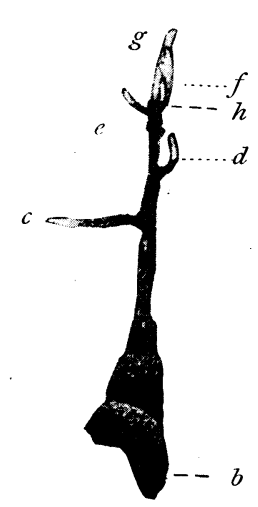

Fig. 7.

Xylaria arbuscula. $b$ Stroma mit den Peritherien; $c, d, e$, $f, g$ reproduzierte Fruchtkörper; $h$ die Stelle, wo der Fruchtkörper vom Substrat entfernt wurde.

Natürl. Größe.

Lebend und reproduktionsfähig bleiben auch die Zellen im Fruchtträger solcher Objekte, die bereits beide Fruktifikationsprozesse durchlaufen haben. Analoges Verhalten, daß - bestimmte Teile eines Fruchtkörpers auch nach Abschluß der Fruktifikation lebende und reproduktionsfähige Zellen konservieren, kennen wir bereits von dem schon mehrfach erwähnten und von Biffen untersuchten Agaricus velutipes.

An den isolierten Teilstücken jüngerer Objekte, worunter ich alle Fruchtkörper vor Anlage des Stromas mit den Askussporen verstehe, vollzieht sich, falls keine hindernden Einflüsse hinzukommen, die reproduktive Tätigkeit an der Schnittfläche. Wird dagegen die Reproduktion an der Schnittfläche von vornherein unmöglich gemacht, so tritt sie an irgend einer Stelle unter der Rindenschicht ein, die in diesem Falle durchbrochen werden muß.

An Teilstücken älterer Objekte mit ausgebildetem Stroma werden meist mehrere Fruchtkörper angelegt, und zwar sowohl auf der Schnittfläche, wie auf den unverletzten Seitenflächen.

Die reproduzierten Fruchtkörper haben mehr oder weniger einen Habitus, wie ihn der Pilz während der Konidienfruktifikation zeigt, und zwar erinnern sie um so mehr an normale Fruchtkörper, je älter das Objekt ist, von dem die Reproduktion ausgeführt wurde.

Im Zusammenhange mit der ausgezeichneten Reproduktionsfähigkeit steht die Regenerationsfähigkeit auf dem natürlichen Substrat stehenbleibender Objekte, die noch nicht fruktifiziert haben, insofern dieselben die äußerste Spitze, die ihnen weggeschnitten wird, vollkommen zu ersetzen vermögen. 
Ähnliche Resultate ergaben die Versuche mit Xylaria hypoxylon. Die Zahl der mit diesem Pilze unternommenen Untersuchungen war aber bei weitem kleiner, weil uns nur solche Objekte zur Verfügung standen, die gerade Konidien abschnürten oder im Begriffe waren, es zu tun. In der üblichen Weise stellte ich wieder durch Querschnitte Teilstücke aus dem Fruchtkörper her, die auf feuchtes Fließpapier gelegt wurden. An allen Versuchsobjekten war schon am Tage nach der Operation eine rege Wachstumstätigkeit zu konstatieren. Das Endresultat dieser Wachstumstätigkeit fiel aber an den verschiedenen Teilstücken sehr verschieden aus, und zwar handelte es sich dabei darum, welcher Partie des Fruchtkörpers das Teilstück entnommen war. An den Schnittflächen der Teilstücke, die dem basalen Teile des Fruchtkörpers angehörten, gingen weiße Hyphenschöpfe hervor, die eine Länge von 2-3 mm erreichten. Auch hier ließ sich nachweisen, daß nur die Markhyphen zu wachsen beginnen, so daß sich die Hyphensprossung scharf gegen die periphere Rindenschicht absetzt. Während aber bei Xylaria arbuscula an Gebilden gleicher Art Konidien abgeschnürt wurden, unterblieb hier die Bildung derartiger Fortpflanzungsorgane. Die Hyphenschöpfe färbten sich mit der Zeit dunkel und gingen mit dem Mutterstück zugrunde.

$\mathrm{Da}$ aber Nährstoffe für weit bedeutendere Leistungen in solchen Teilstücken vorhanden sind, lehrt ein anderer Versuch. Nimmt man die Hyphenschöpfe am zweiten Tage nach ihrer Entstehung durch einen Querschnitt weg, der ungefähr $2 \mathrm{~mm}$ hinter der ursprünglichen Schnittfläche einsetzt, so lassen die nunmehr frei gelegten Hyphen nach zwei Tagen neue Schöpfe hervorgehen, die die Länge der zuerst angelegten erreichen. Diesen Versuch kann man fortsetzen, bis das Teilstück abstirbt.

Die jüngeren Teilstücke, mehr dem Scheitel entnommen, besaßen dagegen eine ausgezeichnete Reproduktionsfähigkeit. Die Neubildungen, die sie hervorbrachten, differenzierten sich zu Gebilden, wie sie während der normalen Entwicklung am Organismus entstehen. So zeigt Fig. 9 mit $b$ ein ausgelegtes Teilstück. Im Kulturgefäße ist ein neues Organ (a) daran entstanden, das den Habitus eines normal wachsenden Fruchtkörpers hat und das eben die charakteristische dichotomische Verzweigung vollzogen hat. Interessant ist das durch Fig. 8 wiedergegebene Objekt. Der ursprüngliche Scheitel liegt in $V$, bei $a$ ist das Objekt vom Muttersproß entfernt worden. An diesem basalen Ende hat sich ein neues Organ gebildet, das durchaus einem jungen Fruchtkörper 
gleicht. Die Neubildung bei Fig. 9 erreicht fast die Länge des Mutterstückes, die bei Fig. 8 bis zwei Drittel derselben.

In derselben Weise wie bei Xylaria arbuscula tötete ich die Wundflächen der Teilstücke in der Flamme ab. Ein Erfolg analog wie bei Xylaria arbuscula, daß die Rindenschicht durch eine seitliche Sprossung durchbrochen wird, blieb aber aus.

Die Untersuchungen ergaben also, daß die Zellen des Fruchtkörpers von Xylaria hypoxylon in ihrer Qualität sehr voneinander abweichen. $\mathrm{Zu}$ wachsen vermögen zwar alle lebendigen Zellen, wenn sie

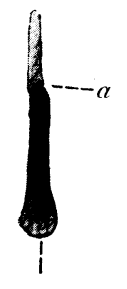

$v$

Fig. 8.

Fig. 9. ron Xylaria hypoxylon. ursprünglichen Vegetationspunkte, $a$ Neubildungen. Natürl. Größe. durch Verwundung dazu angeregt werden. Eine Reproduktion kann aber nur von den jüngeren Zellen in der Nähe des Scheitels ausgeführt werden, den älteren Zellen in der Gegend der Stielbasis fehlt die Fähigkeit dazu. Bei Xylaria hypoxylon liegen also die reproduktionsfähigen Zellen in der Nähe des Vegetationspunktes, den dieser Pilz besitzt. $\mathrm{Da}$ a aber mit dem Vorhandensein eines Vegetationspunktes nicht ein qualitativer Unterschied zwischen älteren und jüngeren Zellen verbunden sein muß, lehren die Versuche mit älteren Fruchtkörpern von Xyl. arbusc.

4. Daedalea unicolor, Polyporus versicolor L., Polyporus caudicinus Schroet. (= Polyporus sulphureus Fr.), Polyporus Braunii.

Daß wir an diesen langsamer wachsenden, meist derberen Pilzen auch jene Reaktionen erwarten durften, wie wir sie an Xylaria, Agaricus und Coprinus kennen lernten, beweist das Verhalten des von Goebel untersuchten Stereum hirsutum ${ }^{1}$ ). Goebel beließ den Pilz auf seinem uatürlichen Substrate im Walde und schnitt große Teile vom Thallus weg. Aus Zeichnung und Beschreibung geht hervor, in welcher Weise der Organismus reagierte. Die Reaktion machte fast den Eindruck einer Regeneration, konnte aber doch nur als Reproduktion angesprochen werden, denn es waren neue Individuen entstanden, von denen jedes seine eigenen charakteristischen Zonen hatte. Von welchen Zellen die Reproduktion der neuen Fruchtkörper ausging, hat Goebel nicht angegeben.

1) Goebel, Flora 1903, pag. $143 \mathrm{ff}$. 
Für meine Untersuchungen wählte ich zunächst Polyporus versicolor. Ich behandelte den Pilz im Freien, indem ich durch Schnitte verschiedene Randteile vom Thallus entfernte. Die Reaktion stimmte mit der von Goebel an Stereum hirsutum beobachteten überein, indem ebenfalls von der Schnittfläche aus neue Fruchtkörper reproduziert wurden. Nur verlief die Bildung dieser Fruchtkörper in einem wesentlich kürzeren Zeitraume. Goebel gibt für Stereum hirsutum eine'Zeit von mehr als einem halben Jahre an. Bei Polyporus versicolor dagegen war schon nach 8 Tagen an dem verletzten Thallus ein bedeutender $\mathrm{Zu}$ wachs entstanden. Als besonders günstig erwies sich ein nach der Operation einsetzender Regen, der das Objekt zu lebhaftem Wachstum anregte.

Schon die makroskopische Betrachtung ließ vermuten, daß an der reproduzierenden Tätigkeit nur die Markhyphen (Innenhyphen) teilnehmen. Die Vermutung wurde dadurch bestätigt, daß ich Thallusteile der verschiedensten Größe, die ich auf feuchtes Fließpapier legte, untersuchte. An diesen Teilstücken wuchsen aus der Wundfäche wulstartige, kallusähnliche Gebilde hervor. Die Annahme, daß sich diese Wucherungen zu Fruchtkörpern differenzierten, bestätigte sich nicht. $\mathrm{Zu}$ einer solchen Leistung fehlt dem isolierten Teilstück die Fähigkeit, jedoch nicht die Nährstoffe; denn nimmt man den kallusartigen Zuwachs durch einen Schnitt weg, so beginnen die nunmehr frei gelegten Zellen auszusprossen. Immerhin reichte das Ergebnis aus, um festzustellen, daß das Wachstum von den Markhyphen ausging. Sicher dürfen wir annehmen, daß auch bei Stereum hirsutum die Reproduktionstätigkeit ihren Sitz in den Markhyphen hat.

Bei den isolierten Teilstücken von Polyporus versicolor ist die Kürze der Zeit bemerkenswert, innerhalb welcher die Reaktion sich vollzieht. Schon nach vier Tagen hatte das Auswachsen seinen Höhepunkt erreicht. Darnach begannen die Objekte abzusterben.

In der gleichen Weise untersuchte ich Daedalea unicolor, konnte aber verhältnismäßig wenig Erfolge verzeichnen. Der verletzte Thallus, der auf seinem Substrat (Kirschbaum) verblieb, [reagierte gar nicht; auf den Schnittflächen der auf Fließpapier ausgelegten Teilstücke erhoben sich nur zerstreut wulstartige Wucherungen. Die gesamte Schnittfläche vermochte nicht daran teilzunehmen. Selbstverständlich kam es auch zu keiner weiteren Differenzierung. Die Reaktion ist nur als ein stellenweise einsetzendes Wachstum zu bezeichnen. Es steht aber auch hier fest, daß die wachstumsfähigen Zellen nur den Markhyphen angehören. 
Weiterhin benutzte ich den an Weidenstämmen häufig auftretenden Polyporus caudicinus Schroet. (= Polyporus sulphureus Fr.). An den Fruchtkörpern dieses Pilzes, die im Freien verblieben, führte ich ebenso wie Massart an Trametes gibbosus ${ }^{1}$ ) senkrechte Schnitte aus, die alle horizontalen Lagen trafen, die den verschiedenen Wachstumsperioden entsprechen. Nach Massarts Angaben scheinen sich bei Trametes gibbosus stets alle Zellen der Schnittfläche an dem auf die Verletzung folgenden Wachstum zu beteiligen, so daß nach Verlauf einer gewissen Zeit von der Wundfläche nichts mehr zu sehen ist. Das ist nach meinen Beobachtungen bei Polyporus candicinus nur dann der Fall, wenn die Schnittfläche geringen Umfang be-

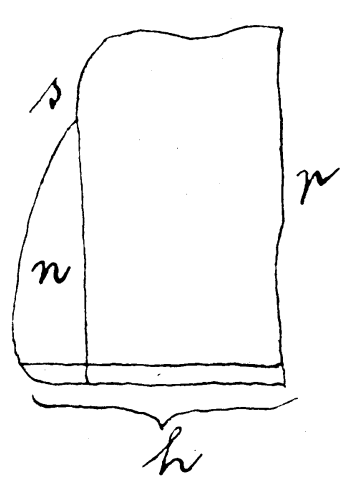

Fig. 10.

Schematischer Querschnitt durch Polyporus candicinus. $n=$ Neuzuwachs, $h=\mathrm{Hy}$ menium, $s \doteq$ Schnittfläche, $r=$ Ansatzfläche des Fruchtkörpers am Baum. Natürl. Größe. sitzt. Wird aber die Schnittfläche so orientiert, wie es oben beschrieben wurde, so unterläßt ein Teil der freigelegten Zellen das Auswachsen, und zwar ist es stets der Teil, der am weitesten vom Hymenium entfernt liegt, also der obere Rand der Schnittfläche - in Fig. 10 mit $s$ bezeichnet. Die Grenze zwischen wachsenden und nicht wachsenden Zellen ist sehr streng gezogen, so daß die Neubildung scharf gegen den oberen Teil der Schnittfläche, der dauernd freiliegt, absetzt. An seiner Unterseite bildet der Neuzuwachs Hymenium $(h)$, das in das Hymenium des Mutterorganismus übergeht.

Offenbar ist die Neubildung als ein neuer Fruchtkörper aufzufassen, der sich bei Polyporus candicinus nur nicht so deutlich abhebt, als es bei unseren Versuchen mit Agaricus campestris der Fall ist.

$\mathrm{Da}$ wir es hier mit einer korrelativen Reaktion zu tun haben, die durch die Verletzung ausgelöst wurde, geht daraus hervor, daß nicht alle Zellen der Schnittfläche, die wachstumsfähig sind, zu wachsen beginnen. Legen wir dagegen einen Fruchtkörper, der in derselben Weise beschnitten ist, auf feuchtes Fließpapier, so beginnen an allen Stellen der Schnittfläche Zellen zu sprossen. Es müssen deshalb innere Einflüsse vorhanden sein, die an einem Fruchtkörper, der auf seinem Substrat verbleibt, nur einen Teil der freigelegten Zellen zum Auswachsen bestimmen. Es liegt hier wieder ein Beispiel vor, daß nicht

1) Massart, l. c. pag. 19- 
immer eine Regeneration ausgeführt wird, wo unter Umständen die Fähigkeit dazu vorhanden ist.

Daß der Pilz sehr schnell zur Reaktion bereit ist, lehrt ein Versuch, den ich am 2. Dezember, also zu einer Zeit, wo die Wachstumsperiode vorüber ist, ausführte. Ich stellte aus einem Fruchtkörper verschieden große Teilstücke her, die ich bei Zimmertemperatur von ca. $20^{\circ} \mathrm{C}$ auf angefeuchtetes Fließpapier auslegte. Schon am 6. Dezember waren deutliche Zellwucherungen auf den Wundfächen zu konstatieren.

An denselben Teilstücken tritt noch ein anderes Phänomen auf. Die graugrüne natürliche Oberfläche des Pilzes gewinnt an den isolierten Objekten nach zwei Tagen ein recht frisches Aussehen. Ein Druck mit dem Finger hinterläßt eine deutlich dunkel gefärbte Spur. Die Betrachtung von Schnitten sagt uns, $d a ß$ auch an diesen unverletzten Oberflächenteilen ein Wachstum begonnen hat. Die äußersten Hyphenenden sind wohl immer, wenigstens zum großen Teile, abgestorben, aber durch die Wärme und die Feuchtigkeit werden die noch dazwischenoder darunterliegenden lebenden Zellen zum Wachstum angeregt; Diese hervorwachsenden Zellen geben der Oberfläche das Aussehen, das ein normaler Fruchtkörper zu Beginn seiner Vegetationsperiode hat.

Endlich untersuchte ich noch einen Pilz, den ich im Warmbause des botanischen Gartens an der Unterseite von feuchten, morschen. Bohlen fand. Er wurde als Polyporus Braunii bestimmt und ist vor allem an der chromgelben Färbung seiner Oberfläche zu erkennen.

Meine Versuche führte ich an Ort und Stelle aus, indem ich durch senkrechte Schnitte Teile von dem mit seiner ganzen Fläche dem Substrate aufsitzenden Fruchtkörper entfernte. Nach Verlauf von acht Tagen hatte der Pilz eine neue Oberfläche auf der Wunde reproduziert, die sich sofort durch ihre chromgelbe Färbung als solche zu erkennen gab. Die mikroskopische Betrachtung zeigte, daß diese Oberfläche Hyphensprossungen, senkrecht zur Schnittfläche, ihren Ursprung verdankte, daß sich also nicht die äußersten, durch die Verletzung freigelegten Hyphen gelb gefärbt hatten.

Teilstücke, im feuchten Raume bei gleicher Temperatur wie im Warmhause gehalten, verloren bald ihre schöne intensive Färbung, und ich konnte nur konstatieren, daß keine der erhofften Reaktionen eingetreten war.

Die Untersuchungen mit diesen Polyporeen lehren wieder, daß trotz ihrer spezifischen Ausbildung die lebendigen Zellen der untersuchten Pilze die Fähigkeit, bei entsprechendem Reiz von neuem die Wachstumstätigkeit aufzunehmen, nicht verloren haben. Reproduktion 
und Regeneration werden aber von diesen Objekten mit Ausnahme von Daedalea unicolor nur dann ausgeführt, wenn sie auf ihrem natürlichen Standorte behandelt werden. Am isolierten Teilstück trat bei unseren -Versuchen, wie das regellose Auswachsen der Zellen auf der Schnittfläche einiger dieser Fruchtkörper andeutet, das korrelative Schaffen und Walten nicht ein, das zu einem reproduktiven oder regenerativen Ersatz des Hinweggenommenen führt.

\section{Claviceps purpurea.}

Die Untersuchung dieses Sklerotiums sollte nicht dazu dienen, das Vorhandensein, embryonaler Zellen oder solcher, die es werden können, festzustellen; denn daß das ganze Sklerotium, mit Ausnahme der toten Zellen der Oberfläche, ein embryonales Gebilde darstellt, sagt schon der normale Entwicklungsgang. Da es aber zum Wesen der embryonalen Zellen gehört, daß sie unter verschiedenen determinierenden Einflüssen verschiedene Entwicklungsbahnen einschlagen können, so mußte es von Interesse sein, zu erfahren, wie sich die Sklerotiumzellen von Claviceps in dieser Hinsicht verhalten.

Unser Versuch reiht sich auch nur an längst bekannte Untersuchungen an. Wie wir bereits wissen, teilt schon de Bary von den Sklerotien der Sklerotinien mit, daß die bloßgelegten Markhyphen einen Wundverschluß bilden, in Nährlösung sogar zu „vegetierendem Fadenmyzel" auswachsen ${ }^{1}$ ). Auch Claviceps soll der ersten Reaktion fähig sein 1). Es wachsen ebenfalls die Markhyphen hervor, die „über die Wundfläche Zweige treiben, welche sich zu einer dünnen Filzdecke verflechten. Die inneren, an das unverwundete Mark grenzenden Schichten dieser Decke bilden sich dann zur neuen Rinde aus, die oberflächlichen vertrocknen und werden bald unkenntlich" ${ }^{1}$ ). Von einem Weiterwachsen der ausgesprossenen Hyphen von Claviceps in Nährlösung berichtet de Bary nichts. - Die Art der Reaktion, die er Zweigbildung nennt, und die darauffolgende Rindenbildung genauer kennen zu lernen, sollte die Aufgabe des folgenden Versuchs sein.

Die Kulturen mit Teilstücken wurden mit ganz besonderer Vorsicht ausgeführt, da die Schnittflächen mit Vorliebe von Schimmelpilzen aufgesucht werden. $\mathrm{Zu}$ diesem Zwecke wusch ich die unversehrten Sklerotien etwa 5-10 Minuten in einer Sublimatlösung $(1: 1000)$ und spülte sie darnach mit sterilisiertem Wasser ab. Sie wurden, um eine Infektion zu vermeiden, im Dampfkasten teils quer, teils längsgeschnitten. Die Teilstücke steckte ich in feuchten Sand, der sich in Kristallisier-

1) de Bary, l. c. pag. 42. 
schalen befand und der an drei aufeinanderfolgenden Tagen im Dampfsterilisator keimfrei gemacht worden war. Über die mit Glasplatten verdeckten Kulturgefäße stülpte ich mit Sublimat ausgewaschene Glasglocken, die auf ebenso behandelten Glasscheiben luftdicht aufsaßen. Auf diese Weise habe ich fast alle Kulturen keimfrei gehalten. Sie standen teils im Wärmezimmer bei ca. $24^{\circ} \mathrm{C}$, teils im Keller, wo durchschnittlich eine Temperatur von $8^{\circ} \mathrm{C}$ herrschte. Die im Keller gehaltenen Kulturen keimten nach der Zeit aus, die $\mathrm{Kühn}^{1}$ ) angibt, also nach etwa 90 Tagen. Es bestätigt sich damit Franks Beobachtung ${ }^{2}$ ), daß zur Bildung von Askussporen .,nicht bloß unversehrte, sondern selbst Stücke von Mutterkörnern (z. B. von Schnecken und dergl. angefressen) fähig“" sind.

Nach drei Wochen untersuchte ich meine Objekte das erste Mal. Sie zeigten dem bloßen Auge keinerlei Veränderung, außer daß sich die Schnittfläche etwas verdunkelt hatte. Aber schon gröbere Schnitte, senkrecht zur Wundfläche ausgeführt, ließen erkennen, daß diese Wundfläche dicht mit länglichen Hyphen besetzt war. Zur genaueren Untersuchung dieser Erscheinung wurden Mikrotomschnitte angefertigt. Die Neubildungen sind ganz auffällig von den normalen Zellen unterschieden. Während das Hyphengeflecht des Sklerotiums aus dickwandigen, polygonalen Zellen besteht, sind die neugebildeten Zellen dadurch charakterisiert, daß sie mit einer dünnen Membran versehen und länglich geformt sind. Sehr oft sind sie auch an ihren Enden angeschwollen. Die Sprossungen waren stets ein bis zwei Zellen lang.

Diese Hyphen gehen aus unverletzten Zellen, die an der Wundfläche liegen, hervor. Ob die Hyphenbildung ein korrelatives Absterben dahinterliegender Zellen zur Folge hatte, konnte ich nicht feststellen. Die nächsten, an die Schnittlläche grenzenden Zellen machten äußerlich nicht den Eindruck irgend einer Veränderung. Dagegen zog sich in einiger Entfernung von der Schnittfläche, parallel zu ihr, ein schwach bräunlichgelber Streifen hin, der damit den Zellen gleicht, die an die Rindenschicht anstoßen. Die Gegend, wo sich dieser Streifen befindet, liegt ziemlich tief im Gewebe, ungefähr 4-7 Zellschichten von der Schnittfläche entfernt. Der Wundreiz pflanzt sich demnach ziemlich weit ins Innere fort.

Die Frage nach dem Zweck des eigenartigen Längenwachstums von seiten der äußersten Zellen drängt sich unwillkürlich auf. Nach de Bary bedeutet es einen vorläufigen Wundabschluß. Dagegen spricht

1) Kühn, Krankheiten der Kulturpflanzen, II. Aufl., pag. 117.

2) Frank, Krankheiten der Pflanzen, 1895, Bd. I, pag. 643. 
aber erstens, daß die Hyphen nicht dicht genug stehen, um eine derartige Aufgabe zu erfüllen, und zweitens die Beobachtung, daß sie entgegengesetzt der Angabe von de Bary auch nach 8 Wochen ihr altes Aussehen bewahren, sich also nicht zu einer Decke verfilzen.

Es ist nicht ausgeschlossen, daß nach Ablauf der Ruhezeit bei gleichzeitigem Vorhandensein einer Nährlösung eine ähnliche Reaktion wie bei den Sklerotien der Sklerotinien eintritt, daß also das Sklerotium in ein vegetatives Stadium übergeht.

Hennings untersuchte auch den Fruchtstiel, der im Frühjahr aus dem Sklerotium hervorbricht ${ }^{1}$ ). Er sah, wie sich an abgebrochenen Stielen „seitlich halbkugelige Köpfchen neu entwickelten“ 1), ein deutlicher Beweis, daß auch dieser Teil des Organismus embryonal tätig sein kann.

\section{Besprechung der Resultate.}

Nachdem eine Zelle ausgewachsen und als Baustein bestimmter Ausbildung dem Organismus eingefügt ist, tritt für gewöhnlich bei ungestörter Vegetation eine weitere Entwicklung nicht ein. Solange aber die Zelle lebendig bleibt, hat sie vielfach die Fähigkeit, unter anderen als den bisherigen Bedingungen eine neue Entwicklungsbahn einzuschlagen, noch nicht verloren ${ }^{2}$ ). Diese Möglichkeit bleibt aber meist nur ,im latenten Zustande vorhanden und tritt nur dann hervor, wenn die gegenseitige Beeinflussung der Zellen aufgehoben wird“" $)$. Unter Umständen geschieht es danr, daß wie bei manchen Schimmelpilzen eine einzelne Zelle oder wie bei Xylaria arbuscula ein Zellkomplex einen neuen Organismus reproduziert. Welch hohen Grad die Differenzierung einer einzelnen Zelle erreichen darf, ohne daß sie die Fähigkeit zur Reproduktion verliert, ist in den vorhergehenden Untersuchungen genügend hervorgetreten. Es liegt infolgedessen die Frage nahe, ob bei den Pilzen gerade so wie bei gewissen Algen und Moosen jede einzelne Zelle die Fähigkeit besitzt, für sich das Ganze zu bilden.

$\mathrm{Daß}$ Zellen, wie die Scheitel- und Gliederzellen von Penicillium glaucum und Aspergillus niger, im isolierten Zustande wachstumstätig bleiben und einen neuen Organismus entstehen lassen können, war zu erwarten, treiben doch auch innerhalb des Verbandes viele Zellen Seitenzweige, aus denen wiederum Seitenzweige hervorgehen, die schließlich Konidienträger entstehen lassen. Abgesehen davon, daß die Zelle isoliert dasselbe leistet wie innerhalb des Verbandes und daß bei Penicillium glaucum die Seitenzweige nicht nur unter oder in der Nähe

1) P. Hennings, l. c. pag. 140.

2) Goebel, Organographie 1898, pag. 35, 36 . 
der oberen Scheidewand, sondern auch über der unteren hervorbrechen, treten an diesen Zellen keine neuen Eigenschaften auf. Es kommt aber noch hinzu, daß eine Anzahl von vegetativen Zellen aus irgend welchen Ursachen das Auswachsen innerhalb des Verbandes unterläßt. Sobald aber solche Zellen isoliert werden, beginnen auch sie auszusprossen, ein Zeichen, daß die Fähigkeit dazu vorhanden war und infolge der Trennung aus dem Verbande realisiert wurde.

Der Gegensatz zwischen potentieller Befähigung und realer Leistung tritt aber noch deutlicher hervor bei den Zellen, die nach ihrer definitiven Ausgestaltung normal nicht mehr wachsen: bei den Zellen der Lufthyphen, des ausgebildeten Konidienträgers von Penicillium glaucum und bei dem Konidienträger von Aspergillus niger, der bereits Konidien abschnürt. Wenn eine dieser Zellen abgetrennt und in Nährlösung gebracht wird, so sproßt sie vegetativ aus. Daß z. B. der Konidienträger von Aspergillus niger das zu allen diesen Tätigkeiten notwendige Keimplasma besitzt, wenn die Bildung der Konidien noch nicht begonnen oder gerade eingesetzt hat, durfte wohl von vornherein angenommen werden. Immerhin war es wichtig, den tatsächlichen Nachweis dafür zu erbringen, daß alle Zellen einer modifizierten Tätigkeit fähig sind; denn es ist bekannt, daß nicht immer allseitig befähigte Zellen die Wachstumstätigkeit aufnehmen können, wie sich z. B. nach den bisherigen Untersuchungen das Sporangium von Phycomyces nitens als unfähig erwies.

Nach alledem läßt sich feststellen, daß in jeder Zelle von Aspergillus niger und Penicillium glaucum, da jede nach der Isolation aus sich den Gesamtorganismus zu erzeugen vermag, „potentiell auch die Gesamtbefähigung, also das gesamte unerläßliche Idioplasma (Keimplasma, Erbmasse)" vorhanden ist ${ }^{1}$.

Schon bei den einzelligen Pilzen Mucor stolonifer und Phycomyces nitens müssen Einschränkungen gemacht werden, insofern es nicht gelungen ist, jeden Zellteil zum Auswachsen zu bringen. Was die Teilstücke vegetativer Hyphen von Mucor stolonifer anbetrifft, so lehrt schon die normale Entwicklung, daß Seitenhyphen an jeder Stelle einer Hyphe angelegt werden können; wo aber solche Aussprossungen an einer Hyphe normal nicht erscheinen, werden sie wie bei Penicillium durch die Isolierung an dem betreffenden Hyphenstück hervorgerufen.

Daß aber ein Lufthyphe von Mucor stolonifer oder ein Sporangiumträger von Phycomyces nitens ebenfalls an allen Stellen zum Aus-

1) Pfeffer, l. c. Bd. II, pag. 176.

Flora 1907 . 
wachsen befähigt bleibt und einen neuen Organismus reproduzieren kann, läßt sich nicht ohne weiteres aus der normalen Entwicklung ableiten, sondern konnte nur durch das Experiment erwiesen werden. Dagegen mußte bei dem Sporangium von Mucor und von Phycomyces vorausgesetzt werden, daß sie potentiell die Fähigkeit zur Reproduktion besitzen, weil beide mit dem für die Bildung der Sporen nötigen Keimplasma ausgestattet sind. Eine Realisierung dieser Fähigkeit gelang aber nur an dem Sporangium von Mucor stolonifer. Es ist aber deshalb noch nicht ausgeschlossen, daß auch noch das Sporangium von Phycomyces nitens durch irgendwelche Umstände zur Reproduktion gebracht wird, wenn nicht bestimmte Ursachen, wie z. B. die Membran, ein Aussprossen verhindert.

Ebenso geben die negativen Erfolge mit dem Sporangiumträger, den Stolonen und Rhizoiden von Mucor stolonifer noch kein Recht, diesen Zellteilen die potentielle Befähigung zum Auswachsen abzusprechen. Nach Brefeld reproduziert z. B. der Sporangiumträger von Mucor Mucedo, wenn er von Piptocephalis befallen wird, neue Sporangienträger, die zu Sporangien anschwellen ${ }^{1}$ ). Dieselbe Befähigung dürfte potentiell auch der Sporangiumträger von Mucor stolonifer besitzen, wenigstens solange durch ihn noch die Stoffe fließen, die im Sporangium gebraucht werden und das Sporangium selbst zum Auswachsen befähigen. Allerdings ist nicht bekannt, welche Ursachen eine Realisierung der angenommenen Fähigkeit hindern könnten.

Bei den Stolonen von Mucor stolonifer gibt schon die normale Entwicklung den Beweis einer vielseitigen Fähigkeit: Sie bilden, wenn sie einen Gegenstand berühren, Rhizoiden, lassen dann Sporangienträger mit Sporangien entstehen und wachsen unter Umständen noch nach einem anderen Punkte weiter. Diese Wachstumstätigkeit beschränkt sich aber nur auf einen geringen Raum an der Spitze. Trotzdem dürfte wohl auch der übrige Protoplast alles das enthalten, was er zum Auswachsen braucht.

Bei den Mucorineen Phycomyces nitens und Mucor stolonifer konnte also die Fähigkeit zur Reproduktion nicht nachgewiesen werden: am Sporangium vom Phycomyces und am Sporangiumträger, den Stolonen und Rhizoiden von Mucor.

Bei dlen höheren Pilzen mit komplizierterem Fruchtkörper waren nicht mehr einzelne isolierte Zellen oder Zellteile, sondern Zellkomplexe Gegenstand der Untersuchung. Wenn infolgedessen von einem solchen

1) Brefeld, 1. c. Heft I, pag. 58. 
Zellkomplex eine Reproduktion ausgeführt wird, so bleibt es trotzdem unentschieden, ob die Fähigkeit dazu schon an und für sich in jeder einzelnen Zelle schlummerte oder ob diese Fähigkeit eine sekundäre Eigenschaft ist, die die Zelle, wie Wiesner und unter Umständen auch Pfeffer ${ }^{1}$ ) annimmt, erst durch Zufluß aus benachbarten Zellen erwirbt. Die Voraussetzung einer Reproduktion sind aber zunächst Zellen, die die Wachstumstätigkeit von neuem aufnehmen können; denn es ist bekannt, daß dies nicht immer einer allseitig befähigten Zelle möglich ist ${ }^{2}$ ). Oft macht eine nicht mehr wachstumsfähige Zellhaut eine Reproduktion unmöglich. Infolgedessen gestatten uns die betreffenden Untersuchungen zunächst ein Urteil darüber, welche Zellen eines Pilzes die Möglichkeit des Wachstums behalten. Zielt die Wachstumstätigkeit auf eine Reproduktion oder Regeneration hin, so muß der Ausgangspunkt in dazu befähigten, embryonalen Zellen liegen. Es ist aber immer zu bedenken, daß eine Reproduktion oder Regeneration nicht schlechthin die gesamte Befähigung der Zellen anzeigt, daß vielmehr stets der Erfolg eines korrelativen Wirkens vorliegt ${ }^{3}$ ).

Von den Zellen der jungen Fruchtkörper von Coprinus ephemerus und Coprinus stercorarius behauptet Brefeld, daß jede einzelne Zelle befähigt sei, einen neuen Organismus zu bilden. Da auch er nur Zellkomplexe verwandte, so ist nicht erwiesen, ob das auch von einer isolierten Zelle gesagt werden kann. Von einer Basidie zwar, die noch keine Sporen abgeschnürt hat, muß wie bei einem Konidienträger von Aspergillus schon von vornherein angenommen werden, daß sie das komplete Keimplasma besitzt und deshalb wohl auch isoliert einen neuen Pilz ergeben dürfte. Eine solche Annahme kann aber nicht mehr von Palissaden-, Stiel- und Hutzellen gemacht werden. Für diese Zellen konnten nur die Versuche den Nachweis erbringen, daß auch sie im Verband mit anderen Zellen befähigt sind, einen neuen Organismus zu reproduzieren. Da die Versuche mit den Zellen aller Gewebe dieser Pilze vorgenommen wurden, so läßt sich wohl behaupten, daß jede lebendige Zelle im jungen Fruchtkörper von Coprinus ephemerus und Coprinus stercorarius wachstumsfähig bleibt und daß sie innerhalb eines Zellkomplexes fähig werden kann, einen neuen Organismus zu bilden.

Die Mitteilungen von Magnus über Agaricus campestris ${ }^{4}$ ), die Beobachtungen von Massart ${ }^{5}$, , die in dieser Arbeit beschriebenen

1) Pfeffer, Physiol., Bd. II, pag. 178.

2) Ders., l. c. Bd. II, pag. 178 u. 205.

3) Pfeffer, l. c. Bd. II, pag. 180.

4) Magnus, l. c. pag. 129 .

5) Massart, 1. c. pag. 18. 
Versuche an demselben Pilze und die Beobachtungen von Hennings an Russula, Tricholoma u. a. ${ }^{1}$ ) deuten daraufhin, daß auch andere Agaricineen aus derartig befähigten Zellen aufgebaut sind. Zu wachsen beginnen eine Anzahl Stielzellen von Agaricus campestris, wenn der Hut vom Stiel getrennt wird, obwohl es zu keiner Reproduktion eines Fruchtkörpers kommt. An einem steril aus Hut oder Stiel herausgeschnittenen Teilstück entsteht ein weißer Überzug, der auf wachsende Zellen zurückzuführen ist. An einem isolierten Stiele kann sogar die Reproduktion eines neuen, wenn auch eigentümlich gebauten Fruchtkörpers ausgeführt werden. Leichte Verletzungen, die durch Tierfraß an der Oberfläche des Hutes entstehen, werden vernarbt. Nach Magnus regeneriert der Hut die künstlich entfernten Lamellen, wenn auch in einer morphologisch etwas abweichenden Form. Alle diese Erscheinungen gestatten uns anzunehmen, daß bei vielen Agaricineen wie bei Coprinus zunächst einmal alle lebendigen Zellen wachstumstätig werden können und daß Zellen des Hutes, des Stieles, des Hymeniums und der Oberfläche die Fähigkeit zur Reproduktion, resp. Regeneration besitzen.

Wachstumsfähige Zellen ließen sich auch an all den übrigen untersuchten Pilzen nachweisen; es hat den Anschein, als ob jeder lebendigen Zelle dieser Pilze die Fähigkeit zukomme, von neuem die Wachstumstätigkeit aufzunehmen. Reproduktion und Regeneration treten aber nicht so unbedingt auf wie bei den Agaricineen. Zwar konnte an allen diesen Pilzen mit Ausnahme von Daedalea unicolor eine Ersatztätigkeit ausgeführt werden, jedoch nur unter besonderen Bedingungen, dazu gehört bei den Polyporeen, daß sie auf ihrem Substrate stehen bleiben, bei Xylaria hypoxylon, daß die Teilstücke dem Scheitelteil des Fruchtkörpers entstammen. Die älteren Zellen von Xylaria hypoxylon vermögen, obwohl auch sie wachstumsfähig bleiben, nicht in den embryonalen Zustand zurückzukehren, welche Fähigkeit dagegen alle lebendigen Zellen von Xylaria arbuscula auch am isolierten Teilstück zeigen.

Es sollte nun weiter erörtert werden, in welcher Weise die Außenbedingungen auf die Lenkung der Ersatztätigkeit von Einfluß sind, und im Zusammenhange damit, inwieweit die Qualität der Neubildung vom Zustande des Mutterorganismus abhängig ist.

Während es unter Blütenpflanzen, Farnen und Moosen Organismen gibt, die bei voller Konstanz der Außenbedingungen ihre spezifische Ontogenese durchlaufen, ist neben den meisten Algen, auch bei den meisten Pilzen eine Veränderung der Außenbedingungen nötig, wenn

1) Hennings, l. c. pag. 138. 
der Entwicklungsgang mit der Produktion von Fortpflanzungsorganen abschließen soll ${ }^{1}$ ). Fraglich ist es, ob das auch für alle höheren Pilze gilt, was von Pfeffer in Zweifel gestellt wird ${ }^{2}$ ). Jedenfalls steht aber soviel fest. daß die meisten Pilze ihren Formenkreis nur bei einem bestimmten Wechsel der äußeren Einflüsse durchlaufen. Die Reizbedingung für den Fortpflanzungsprozeb kann aber gegeben werden durch Abnahme der Nahrung, Ansammlung von Stoffwechselprodukten oder durch Übergang in ein anderes Medium. Werden dagegen die Außenbedingungen konstant erhalten, so erfolgt ein rein vegetatives Wachsen und Vermehren. Der Experimentator hat es also in der Hand, durch Veränderung der entsprechenden äußeren Faktoren die auf Bildung der Fortpflanzungsorgane hinzielenden Wachstumsvorgänge auszulösen oder durch Konstanz der äußeren Einflüsse den Pilz im vegetativen Zustande beharren zu lassen.

Die Anpassungsfähigkeit vieler Pilze geht aber noch weiter. Man kann nämlich ein Organ (Zelle, Zellteil), das durch Veränderung der äußeren Faktoren entstand, wieder unter die Außenbedingungen versetzen, bei deren Konstanz nur vegetatives Wachstum vor sich geht. Das geschieht aber dadurch, daß man die betreffenden Objekte, die durch Anhäufung von Stoffwechselprodukten im Nährsubstrat, durch Nahrungsmangel oder durch Herauswachsen aus dem Nährsubstrat entstanden, wieder in frische Nährlösung, resp. auf frisches Nährsubstrat bringt, so daß sie von diesem unmittelbar umgeben werden. Ist die Ausgestaltung der betreffenden Objekte noch nicht zu weit fortgeschritten, so ist in einer Anzahl von Fällen eine Umlenkung der bisherigen Entwicklungsrichtung möglich, und es beginnt von neuem vegetatives Wachstum.

Auf diese Weise war es möglich, aus einem Sporangiumträger von Phycomyces nitens, aus einem Konidienträger von Aspergillus niger, aus einer Konidienträgerzelle von Penicillium glaucum ein vegetatives Myzel zu erzielen. Auch Fruchtkörperteile einiger höherer Pilze besitzen die Fähigkeit, aufs vegetative Stadium zurückzukehren, wie es durch das Verhalten von Coprinus ephemerus, Coprinus stercorarius und Peziza sklerotiorum dargetan wird. Eine ebensolche Umlenkung gelingt mit den Sklerotien von Coprinus stercorarius und den Sklerotien der Sklerotinien.

Andere Organe, die unabhängig vom Fortpflanzungsprozesse entstehen, nämlich die Lufthyphen von Penicillium glaucum, Aspergillus

1) Vergl. Klebs, Jahrb. f. wiss. Bot. 1900, Bd. XXXV, pag. 80.

2) Pfeffer, l. c. Bd. II, pag. 250. 
niger, Phycomyces nitens und Mucor stolonifer vermögen in derselben Weise zu reagieren, indem sie in Nährlösung zu Myzel auswachsen.

Der bei all den genannten Objekten einheitliche Verlauf der Reaktion erfährt eine Ausnahme durch das Verhalten des Sporangiums von Mucor stolonifer. Wurde dieses Sporangium untergetaucht, so bildeten sich zwar auch Keimschläuche, aber dadurch, daß diese allesamt sofort über das Nährsubstrat hervorwachsen und an ihren Enden Sporangien entstehen lassen, wird angezeigt, daß die veränderte Tätigkeit im Sporangium darnach strebt, auf dem kürzesten Wege von neuem Fortpflanzungsorgane zu produzieren. Diese Beobachtung gestattet aber noch nicht anzunehmen, daß das Sporangium nie Myzel bildet wird. Eine solche Annahme wird schon durch das Auswachsen des Sporangiums hinfällig. Außerdem genügen die beiden Resultate, die erzielt wurden, noch nicht, um das beobachtete Verhalten zu generalisieren. Auch an der Zygospore von Mucor Mucedo entsteht bei den gleichen Außenbedingungen in den meisten Fällen ein Keimschlauch, der sofort zur Fruchthyphe wird ${ }^{1}$ ). Doch ist ebenfalls, wenn auch seltener beoachtet worden, daß die Zygospore ein Myzel bildet.

Es ergibt sich so, daß bei einer Anzahl von Pilzen reproduktionsfähige.Zellen oder Zellteile, die nicht dem Myzel angehören, unter Außenbedingungen, die einer myzelialen Entwicklung günstig sind, zu vegetativen Hyphen auswachsen, also Gebilde hervorbringen, die ihrer Qualität nach nicht mit dem Zustande des reproduzierenden Objektes übereinstimmen. Eine Ausnahme macht nach den bisherigen Erfahrungen das Sporangium von Mucor stolonifer, das trotz der für ein myzeliales Aussprossen günstigen Außenverhältnisse, kein Myzel bildet, sondern sofort neue Sporangien entstehen läßt, also Neubildungen produziert, die seiner eigenen Qualität entsprechen.

Mit diesen Betrachtungen sind wir gleichzeitig der Frage der vegetativen Vermehrung näher getreten. Sie wird für Pilze erst vollständig gelöst sein, wenn man in der Kenntnis der künstlichen Nährböden weiter vorgedrungen ist. Nach den bisherigen Erfahrungen darf man wohl annehmen, daß dort, wo reproduktionsfähige Zellen nachgewiesen wurden, auch eine vegetative Vermehrung möglich sein wird.

Die größte Anzahl der Versuche wurde nun aber so angeordnet, daß keine Veränderung in den Außenverhältnissen eintrat. Vegetatives Myzel blieb in seiner Nährlösung, Fruchtkörper höherer Pilze wurden auf ihrem natürlichen Nährboden behandelt. Wenn auch nicht bei

1) Brefeld, l. c. Heft I, pag. 23. 
allen Pilzen nachgewiesen ist, daß Nahrungsmangel den Anstoß zur Bildung der Fortpflanzungsorgane gibt, so wird doch bei den meisten dieser Faktor maßgebend sein; auf jeden Fall wirkt er auf Teilstücke von Fruchtkörpern, die so ausgelegt werden, dạ eine Nahrungszufuhr aus der Umgebung ausgeschlossen ist. Unter diesen Umständen war der Erfolg der im Teilstück durch die Isolierung ausgelösten Wachstumstätigkeit derselbe wie bei vielen Myzelien, in deren Umgebung Abnahme der Nahrung eintritt: Es wird ein Fruchtkörper angelegt; vorausgesetzt natürlich, daß dazu befähigte Zellen vorhanden sind. Dieselbe Reaktion tritt aber auch meist an Fruchtkörpern, die auf ihrem Substrat stehen bleiben, dann ein, wenn die Verletzung so stark ist, daß dem Objekt eine Weiterentwicklung in der angegebenen Richtung unmöglich wird. Es erfolgt ebenfalls die Reproduktion eines Fruchtkörpers. Offenbar rettet der Organismus, wie Goebel interpretiert, „unter Umständen, welche der Entwicklung ungünstig sind, daß „Keimplasma“ auf möglichst kurzem Wege" 1 ).

Eine Regeneration wird an den Fruchtkörpern dann ausgeführt, wenn der Eingriff nicht allzu störend wirkt, wenn z. B. durch Tierfraß Teile der Oberfläche oder des Hymeniums weggefressen werden.

Nun gibt es noch eine Anzahl von Fällen, wo zwar die Bedingungen für eine Regeneration günstig sind, wo aber doch eine Reproduktion vom Organismus vorgezogen wird. Brefeld konnte z. B. eine Regeneration des Hutes von Coprinus stercorarius nur dann erzielen, wenn er die Reproduktion von Fruchtkörpern auf dem Sklerotium verhinderte, und nach Massart erfolgt ebenfalls am Fruchtkörper von Agaricus campestris eine Regeneration gewisser Teile nur bei Unterdrückung der angestrebten Reproduktion. Diese an anderen Pflanzen schon oft beobachtete Erscheinung ist nach Pfeffer so zu verstehen, daß eine korrelative Hemmung der Regeneration von der Ersatztätigkeit ausgeht, „die in der Pflanze augenscheinlich zunächst aufgenommen und bevorzugt wird und tatsächlich oft ökologisch vorteilhafter ist" "2).

Was aber die Qualität der Neubildungen anbetrifft, so hängt sie, falls ein Objekt unter den Außenverhältnissen bleibt, unter denen es sich normal entwickelt, von dem Stadium ab, in dem sich der Organismus befindet ${ }^{3}$ ). Am Myzel wird Myzel gebildet, am Sporangiumträger von Mucor Mucedo und Phycomyces nitens von neuem Sporangiumträger mit Sporangien. Fruchtkörperteile reproduzieren neue Fruchtkörper.

1) Goebel, Organographie, 1898, pag. 122.

2) Pfeffer, l. c. Bd. II, pag. 208.

3) Goebel, Morphologische und biolog. Bemerkungen, Flora 1903, pag. 145. 
Auf ihrem Substrat stehen bleibende Fruchtkörper regenerieren entweder das Hinweggenommene oder lassen ebenfalls neue Fruchtkörper entstehen.

Die Bedingungen des Absterbens mycelialer Zellen von Aspergillus niger.

Wenn es auch nicht möglich ist, die letzte Ursache im Protoplasten, durch die der Tod herbeigeführt wird, festzustellen ${ }^{1}$ ), so muß doch versucht werden, das Absterben auf innere oder äußere Faktoren zurückzuführen 2). Es ist demnach zu untersuchen, ob sich der Tod von Myzelzellen bei Aspergillus niger selbstregulatorisch im Verlaufe der Ontogenese einstellt oder ob er durch Außenbedingungen veranlaßt wird.

Pantanelli hat für gewisse Stadien der Entwicklung von Aspergillus niger angegeben, in welchem Verhältnis die Zahl der toten Zellen zu der der lebenden steht $^{3}$ ). Darnach leben die meisten Zellen nicht länger als 4-5 Tage; denn ,schon am 4.-5. Entwicklungstage erscheinen die früher ausgegliederten Zellen auch in isolierten Flocken tot und mit der Verdichtung zu einer Decke bleiben meist nur die oberflächlichen und die Randhyphen am Leben". Da nach Verlauf dieser Zeit auch die Sporenproduktion vollendet ist und nach der Sporenproduktion lebende Zellen nur eine Ausnahme bilden, so liegt es nahe, den Schluß zu ziehen, daß mit Abschluß der Sporenbildung korrelativ die Lebenstätigkeit der vegetativen Zellen aufhört. Es wäre auch verständlich, daß sich bis zu diesem Punkte der Entwicklung die Zelle so abgenutzt hat, daß ihr ein Weiterleben unmöglich wird. Nach einer solchen Interpretation würden es also innere Ursachen sein, die das Absterben der Myzelzellen bedingen.

Besteht aber wirklich eine solche Korrelation? Daß sie nicht bestehen muß, lehren die Erfahrungen mit Xylaria arbuscula und Agaricus velutipes, deren Fruchtkörper noch lange Zeit nach der Abschnürung lebende Zellen enthalten. Es erhebt sich infolgedessen die Frage, ob die Myzelzellen von Aspergillus niger tatsächlich gleichzeitig mit der Sporenbildung absterben.

Zur Beantwortung dieser Frage kultivierte ich den Pilz unter denselben Bedingungen wie Pantanelli. Ich stellte deshalb eine Nährlösung von folgender Zusammensetzung her: Auf $100 \mathrm{ccm} \mathrm{H}_{2} \mathrm{O}-1 \mathrm{~g}$

1) Pfeffer, l. c. Bd. II, pag. 283.

2) Ders., l. c. Bd. II, pag. 283.

3) Pantanelli, Jahrb. f. wiss. Bot., Bd. XL, 1904, pag. 308. 
$\mathrm{NH}_{4} \mathrm{NO}_{3}, 5 \mathrm{~g}$ Traubenzucker, $0,5 \mathrm{~g} \mathrm{KH}_{2} \mathrm{PO}_{4}, 0,25 \mathrm{~g} \mathrm{MgSO}_{4}(0,52 \mathrm{~g}$ $\mathrm{MgSO}_{4}+7 \mathrm{aq}$ ), 0,02 g ZnSO $4,0,001 \mathrm{~g} \mathrm{Fe}_{2} \mathrm{Cl}_{6}$, Spuren von $\left.\mathrm{H}_{3} \mathrm{PO}_{4}{ }^{1}\right)$.

Am dritten Tage nach der Impfung konnte ich beobachten, daß schon einige Hyphen fruktifizierten. Dieses Stadium wählte ich zunächst für meine Untersuchungen aus. Das Hyphengeflecht ist noch so locker, daß sich die einzelnen Hyphen, die einen Konidienträger angelegt hatten, bequem verfolgen ließen. Derartige Myzelflöckchen habe ich sehr oft und mit größter Peinlichkeit nach toten Zellen durchsucht, konnte aber nur konstatieren, daß auch an den Hyphen, die fruktifiziert hatten, tote Zellen eine Ausnahme bildeten ${ }^{2}$ ). Weitaus die meisten Hyphen setzten sich aus lebenden Zellen zusammen. Nun ist aber noch einzuwenden, daß Kulturen auf diesem Stadium die Sporenbildung noch gar nicht abgeschlossen haben; denn es finden sich noch zahlreiche unentwickelte Konidienträger. Ich habe aber meine besondere Aufmerksamkeit den Zellsträngen gewidmet, denen ein vollkommen ausgebildeter Konidienträger angehörte, der selber seine Konidien schon wieder zum großen Teile verloren hatte. In seltenen Fällen entdeckte ich tote Gliederzellen.

Am 4. und 5. Tage treten die toten Zellen in der Weise auf, wie es Pantanelli festgestellt hat. Lebendige Zellen lassen sich aber bis zum 12. Tage nachweisen, beschränken sich jedoch nach Angaben Pantanellis, die ich bestätigen konnte, auf die oberflächlichen und die Randhyphen ${ }^{3}$ ).

Durch das Ergebnis unserer Untersuchung ist die Annahme hinfällig geworden, daß Sporenbildung und Absterben der Myzelzellen zeitlich zusammenfallen. Nun kann aber noch geltend gemacht werden, daß der Vorgang der Fruktifikation die vegetativen Zellen so in Anspruch nimmt, daß ihre Lebensdauer verkürzt wird. Infolgedessen war zur endgiltigen Lösung des Problems noch die experimentelle Behandlung einer anderen Frage nötig: Vermag nämlich eine Unterdrückung der Fruktifikation die Lebensdauer der Zellen zu verlängern?

Die Sporenbildung, die nur bei genügender Transspiration möglich ist, kann dadurch unterdrückt werden, daß der Pilz submers ge-

1) Pantanelli, 1. c. pag. 306 .

2) Um lebende von toten Zellen zu unterscheiden, benutzte ich verschiedene Methoden: entweder plasmolysierte ich mit $10 \%$ Kalisalpeter, oder ich färbte die Objekte mit Methylenblau. Mit der Zeit stellt sich aber eine solche Übung ein, daß man derartige Mittel gar nicht mehr braucht. Trotzdem wandte ich, auch wenn ich glaubte, durch bloße Betrachtung unter dem Mikroskop ein sicheres Urteil erhalten zu haben, eine der beiden Methoden nachträglich an.

3) Pantanelli, l. c. pag. 308. 
züchtet wird. Solche Kulturen erlangt man bequem dadurch, daß man, wie es Klebs getan hat, oberflächlich entstandene Myzeltlöckchen in Nährlösung versenkt. Um von vornherein zu vermeiden, daß das Myzel in Berührung mit der freien Luft kam, benutzte ich folgendes Verfahren: Ich verwandte wieder die Lösung, deren Zusammensetzung ich oben angegeben habe, und gewann durch Beimengung von Agar ein festes Substrat. Dieses Nährsubstrat erwärmte ich in einem Kölbchen solange, bis es sich verflüssigte, impfte es mit Sporen, die sich durch Schütteln so in der Flüssigkeit verbreiteten, daß jeder mit der Platinnadel herausgenommene Tropfen mindestens eine Spore enthielt. Einen solchen Tropfen übertrug ich auf einen sterilisierten Objektträger, auf dem er erstarrte. Da sich der Agar in Flüssigkeit von seiner Unterlage ablöst, mußte eine besondere Vorrichtung angebracht werden, um dies zu vermeiden.

Ich schnitt dünne Glasstreifen (etwa $2 \mathrm{~cm}$ lang und $0,4 \mathrm{~cm}$ breit), die ich, nachdem ich sie durch Hitze sterilisiert hatte, jüber den Agartropfen legte und mit Siegellack befestigte. Den Objektträger brachte ich, mit dem Nährtropfen nach unten, in eine Kristallisierschale. legte ihn auf der einen Seite auf einen Glasblock, um dem späteren Myzel ein Aufsteigen der entstehenden Gasblasen zu erleichtern. Dann goß ich in das Kulturgefäß soviel Nährlösung, bis der Objektträger vollständig bedeckt war. Die Zusammenstellung des Versuches geschah, um eine Infektion zu vermeiden, im Dampfkasten.

Wenn nun doch einige Sporen ihren Weg an die Oberfläche fanden, so wurde das hier entstandene Myzel mit einer sterilisierten Pinzette entfernt.

Nach drei Tagen untersuchte ich die submers entstandenen Kulturen mit Hilfe der angegebenen Methoden auf tote Zellen. Alle Zellen erwiesen sich als lebendig. Am 4. Tage dagegen traten in allen Kulturen abgestorbene Gliederzellen auf, deren Zahl mit dem כ. Tage zunahm. Die Myzelzellen gingen von der Mitte aus zu Grunde, während die Randzellen lebendig blieben. Also sterben auch hier die am frühesten abgegliederten Zellen zuerst ab, wie es Pantanelli an den normalen Kulturen nachgewiesen hat ${ }^{1}$ ). Am 6. Tage haben sich die toten Zellen wieder etwas vermehrt, die Peripherie der Myzelien besteht dagegen durchweg aus lebendigen Zellen. Doch muß noch hinzugefügt werden, daß die Zunahme der toten Zellen nicht regelmäßig vor sich geht. In gleichgroßen und gleichalten Myzelien herrschen bedeutende

1) Pantanelli, l. c. pag. 308 . 
Unterschiede, so daß es nicht möglich ist, ein bestimmtes Verhältnis zwischen lebenden und toten Zellen für gewisse Zeiten anzugeben.

Das Resultat des zweiten Versuchs ist also folgendes: Am 4. Tage der Entwicklung treten in den submers gehaltenen Myzelien die ersten abgestorbenen Zellen auf, und zwar trifft der Tod zunächst die Zellen, die zuerst abgegliedert worden sind. Daraus ergibt sich aber, daß das Alter der submers gehaltenen Zellen das der normal lebenden nicht ïbersteigt. Trotz der Ausschaltung der Sporenbildung tritt also der Tod ebenfalls am 4. bis 5. Entwicklungstage ein. Auch bei einjährigen Pflanzen wird die Lebensdauer der somatischen Teile nur bis zu einem gewissen Grade verlängert, wenn man Blühen und Fruchten verhindert ${ }^{1}$ ). - Der Tod der vegetativen Zellen von Aspergillus niger würde demnach selbstregulatorisch durch innere Ursachen herbeigeführt.

Es fragt sich aber weiter, ob nicht auch äußere Faktoren hierbei wirksam sind.

$\mathrm{Da}$ gewisse Außenbedingungen ein früheres Absterben der vegetativen Zellen bewirken können, lehrt die Untersuchung einer Agarkultur. Ich ließ eine solche Kultur, die kreisförmig wuchs, einen Durchmesser von $3,7 \mathrm{~cm}$ gewinnen, wozu eine Zeit von 7 Tagen nötig war. Der äußerste Rand bestand aus Hyphen, die noch nicht fruktifiziert hatten. Schon unter ihnen traten hin und wieder tote Zellen auf. Die nächste Zone besaß eine Breite von $0,2-0,3 \mathrm{~cm}$ und bestand aus einem Myzel, das erst wenig Fruchtträger ausgebildet hatte, trotzdem eine große Zahl abgestorbener Zellen enthielt. Weiter nach dem Inneren der Kultur stieg die Zahl dieser Zellen rapid, schließlich waren lebende Zellen nur noch an der Oberfläche zu finden.

Als Ursache, die den Tod von Zellen in solchen Hyphen herbeiführt, die noch gar nicht fruktifiziert haben, kommt zunächst die beschränkte Sauerstoffzufuhr in Betracht. Sobald sich einige Hyphen übereinanderlagern, werden die $\mathrm{zu}$ unterst liegenden vom Sauerstoff der Luft abgeschnitten. Ein weiterer Faktor, der die Lebensdauer der dem Substrat aufliegenden Zellen verkürzt, ist wahrscheinlich die Ansammlung schädlicher Stoffwechselprodukte. So läßt es sich erklären, daß der Tod bei den meisten Zellen dieser Agarkulturen früher eintritt als in den vorher untersuchten Myzelien. - Wenn auch nicht in demselben Grade, so werden dieselben Außenbedingungen doch auch bei den Myzelien auf oder in Nährlösung von Einfluß sein. Das beweist die Tatsache, daß einzelne lebende Zellen bis zum 12. Entwick-

1) Pfeffer, l. c. Bd. II, pag. 285. 
lungstage an der Oberseite von oberflächlich entstandenen Myzelien nachgewiesen werden können.

Das Resultat aller Versuche läßt sich in folgender Weise zusammenfassen :

Die Lebensdauer der vegetativen Zellen von Aspergillus niger, der auf Nährlösung gezogen wird, beträgt durchschnittlich 4-う̃ Tage. Das Absterben der Zellen und die Sporenproduktion fallen zeitlich nicht zusammen, auch vermag die Unterdrückung des Fortpflanzungsprozesses die Lebensdauer der Zellen nicht zu verlängern. Die Tatsache, daß lebende Zellen noch bis zum 12. Entwicklungstage an der Oberfläche der Kulturen zu finden sind, läßt annehmen, daß äußere Faktoren, nämlich beschränktere Sauerstoffzufuhr und Ansammlung schädlicher Stoffwechselprodukte den Tod der Zellen früher herbeiführen, als er selbstregulatorisch eintreten würde. 